\title{
QUASILINEAR ELLIPTIC PROBLEM WITHOUT AMBROSETTI-RABINOWITZ CONDITION INVOLVING A POTENTIAL IN MUSIELAK-SOBOLEV SPACES SETTING
}

\author{
SOUFIANE MAATOUK AND ABDERRAHMANE EL HACHIMI
}

\begin{abstract}
In this paper, we consider the following quasilinear elliptic problem with potential

$$
(P) \begin{cases}-\operatorname{div}(\phi(x,|\nabla u|) \nabla u)+V(x)|u|^{q(x)-2} u=f(x, u) & \text { in } \Omega, \\ u=0 & \text { on } \partial \Omega,\end{cases}
$$

where $\Omega$ is a smooth bounded domain in $\mathbb{R}^{N}(N \geq 2), V$ is a given function in a generalized Lebesgue space $L^{s(x)}(\Omega)$, and $f(x, u)$ is a Carathéodory function satisfying suitable growth conditions. Using variational arguments, we study the existence of weak solutions for $(P)$ in the framework of Musielak-Sobolev spaces. The main difficulty here is that the nonlinearity $f(x, u)$ considered does not satisfy the well-known Ambrosetti-Rabinowitz condition.
\end{abstract}

\section{INTRODUCTION}

Let $\Omega \subset \mathbb{R}^{N}(N \geq 2)$ be a bounded smooth domain. Assume that $\phi: \Omega \times[0,+\infty) \rightarrow[0,+\infty)$ is a Carathéodory function such that for all $x \in \Omega$, we have

$$
(\phi)\left\{\begin{array}{l}
\phi(x, 0)=0, \quad \phi(x, t) \cdot t \text { is strictly increasing, } \\
\phi(x, t) . t>0, \forall t>0 \text { and } \phi(x, t) . t \rightarrow+\infty \text { as } t \rightarrow+\infty .
\end{array}\right.
$$

In this paper, we study the following quasilinear elliptic problem

$$
(P) \begin{cases}-\operatorname{div}(\phi(x,|\nabla u|) \nabla u)+V(x)|u|^{q(x)-2} u=f(x, u) & \text { in } \Omega, \\ u=0 & \text { on } \partial \Omega,\end{cases}
$$

where $V$ is a potential belonging to $L^{s(x)}(\Omega), q$ and $s: \bar{\Omega} \rightarrow(1, \infty)$ are continuous functions and $f: \Omega \times \mathbb{R} \rightarrow \mathbb{R}$ is a Carathéodory function which satisfies some suitable growth conditions. Precise conditions concerning the functions $q, s, f$ and $V$ will be given hereafter.

Problem $(P)$ appears in many branches of mathematical physics and has been studied extensively in recent years. From an application point of view, this problem has its backgrounds in such hot topics as image processing, nonlinear electrorheological fluids and elastic mechanics. We refer the readers to [10, 34] and the references therein for more background of applications. In particular, when $\phi(x, t)=t^{p(x)-2}$, where $p$ is a continuous function on $\bar{\Omega}$ with the condition $\min _{x \in \bar{\Omega}} p(x)>1$, the operator involved in $(P)$ is the $p(x)$-Laplacian operator, i.e. $\Delta_{p(x)} u:=\operatorname{div}\left(|\nabla u|^{p(x)-2} \nabla u\right)$. This differential operator is a natural generalization of the $p$-Laplacian operator $\Delta_{p} u:=\operatorname{div}\left(|\nabla u|^{p-2} \nabla u\right)$ where $p>1$ is a real constant. Note that the $p(x)$-Laplacian operator possesses more complicated nonlinearities than the $p$-Laplacian operator (for example, it is nonhomogeneous), so more complicated analysis has to be carefully carried out.

The interest in analyzing this kind of problems is also motivated by some recent advances in the study of problems involving nonhomogeneous operators in divergence form. We refer for instance to the results in 1, 4, 5, 6, 7, 8, 16, 21, 35, 26, 36. The studies for $p(x)$-Laplacian problems have been extensively considered by many researchers in various ways (see e.g. [1, 17, 23, 26]). It should

2010 Mathematics Subject Classification. Primary 35A15, 35B38, 35J62 . 
be noted that our problem $(P)$ enables the presence of many other operators such as double-phase and variable exponent double-phase operators.

Before moving forward, we give a review of some results related to our work. We start by the case where the potential $V \equiv 0$ on $\Omega$. Fan and Zhang in [17, proved the existence of a nontrivial solution and obtained infinitely many solutions for a Dirichlet problem involving the $p(x)$-Laplacian operator. Mihailescu, Pucci and V. Radulescu in [9], have studied the question of multiplicity of solutions for a class of anisotropic elliptic equations with variable exponent. In the anisotropic setting we also refer the reader to [28, 29]. Clément, García-Huidobro and Schmitt in [12, established the existence of a nontrivial solution for more general quasilinear equation in the framework of Orlicz-Sobolev spaces, in the case where the function $\phi$ considered in $(P)$ is independent of $x$, i.e. $\phi(x, t)=\phi(t)$. Remaining in the Orlicz-Sobolev space setting, Bonanno, Molica Bisci and Rădulescu in [5, 6, 7] proved some important results concerning the existence of infinitely many weak solutions for a nonhomogeneous eigenvalue Dirichlet problem (see also [8]). Liu and Zhao in [27, obtained the existence of a nontrivial solution and infinitely many solutions for a quasilinear equation related to problem $(P)$ in the framework of Musielak-Sobolev spaces (see also [16]).

In the above mentioned papers, the authors assumed, among other conditions, that the nonlinearity $f$ satisfy to the well-known Ambrosetti-Rabinowitz condition ((A-R) condition for short); which, for the $p$-Laplacian operator, asserts that there exist two constants $M>0$ and $\theta>p$, such that

$$
0<\theta F(x, t) \leq f(x, t) t, \quad \forall|t| \geq M,
$$

where $F(x, t)=\int_{0}^{t} f(x, s) d s$. Clearly, this condition implies the existence of two positive constants $c_{1}, c_{2}$ such that

$$
F(x, t) \geq c_{1}|t|^{\theta}-c_{2}, \quad \forall(x, t) \in \Omega \times \mathbb{R} .
$$

This means that $f$ is $p$-superlinear at infinity in the sense that

$$
\lim _{|t| \rightarrow+\infty} \frac{F(x, t)}{|t|^{p}}=+\infty .
$$

This type of condition was introduced by Ambrosetti and Rabinowitz in their famous paper [2] and has since become one of the main tools for finding solutions to elliptic problems of variational type; especially in order to prove the boundedness of Palais-Smale sequence of the energy functional associated with such a problem. Unfortunately, there are several nonlinearities which are $p$-superlinear but do not satisfy the (A-R) condition. For instance, if we take $f(x, t)=|t|^{p-2} t \ln (1+|t|)$, then we can check that for any $\theta>p, F(x, t) /|t|^{\theta} \rightarrow 0$ as $|t| \rightarrow+\infty$. However, many recent types of research have been made to drop the (A-R) condition (see e.g. [4, 11, 21, 26] and references therein).

In [4, the authors studied a similar problem as that in [12] and proved the existence of at least a nontrivial solution under the following assumptions on the nonlinearity $f$ : there exist an $N$-function $\Gamma$ (cf. [33]) and positive constants $C, R$ such that

$$
\Gamma\left(\frac{F(x, t)}{|t|^{\phi_{0}}}\right) \leq C \bar{F}(x, t), \quad \forall(x,|t|) \in \Omega \times[R,+\infty),
$$

and

$$
\lim _{|t| \rightarrow+\infty} \frac{f(x, t)}{|t|^{\phi^{0}-1}}=+\infty, \quad \lim _{|t| \rightarrow 0} \frac{f(x, t)}{|t| \phi(t)}=\lambda,
$$

where $\bar{F}(x, t):=f(x, t) t-\phi^{0} F(x, t), \lambda$ some nonnegative constant and $\phi_{0}, \phi^{0}$ are defined in relation (2.1) below (when $\phi(x, t)=\phi(t)$ independent of $x$ ) with specific assumptions. It should be noted that the condition (1.3) is a type of "nonquadraticity condition at infinity", which was 
first introduced by Costa and Magalhães in [13] for the Laplacian operator (with $\phi_{0}=\phi^{0}=2$ ) as follows:

$$
\liminf _{|t| \rightarrow+\infty} \frac{\bar{F}(x, t)}{|t|^{\sigma}} \geq a>0
$$

holds for some $\sigma>0$. We would also like to mention that this condition plays an important role in proving the boundedness of Palais-Smale sequences.

In [11] also, the authors considered a similar problem as that in [12] and proved the existence of a nontrivial solution under the following assumptions on the nonlinearity $f$ : there exist $\mu_{1}, \mu_{2}>0$ such that

$$
\begin{gathered}
\lim _{|t| \rightarrow+\infty} \frac{F(x, t)}{\left.|t|\right|^{0}}=+\infty, \quad \lim _{|t| \rightarrow 0} \frac{f(x, t)}{|t|^{\phi^{0}-1}}=0, \\
\bar{F}(x, t) \leq \bar{F}(x, s)+\mu_{1}, \quad \forall(x, t) \in \Omega \times(0, s) \text { or } \forall(x, t) \in \Omega \times(s, 0),
\end{gathered}
$$

and

$$
\bar{H}(t s) \leq \bar{H}(t)+\mu_{2}, \quad \forall t \geq 0 \text { and } s \in[0,1],
$$

where $\bar{H}(t):=\phi^{0} \Phi(t)-\phi(t) t^{2}$ with $\Phi(t)=\int_{0}^{t} \phi(s) s d s$.

On the other hand, in the few last years, studies on double phase problems have attracted more and more interest and many results have been obtained. Especially, in 21] the authors proved the existence of a nontrivial solution and obtained infinitely many solutions for a double phase problem without (A-R) condition. More precisely, they considered the problem $(P)$ (with $V \equiv 0$ ) with the function $\phi(x, t)=t^{p-2}+a(x) t^{q-2}$, where $a: \bar{\Omega} \mapsto[0,+\infty)$ is Lipschitz continuous, $1<p<q<N$, $\frac{q}{p}<1+\frac{1}{N}$ and the nonlinearity $f$ satisfies the assumptions (1.5) and (1.6) above with $\phi_{0}=p$ and $\phi^{0}=q$. In [20] however, the authors considered the same previous problem and proved the existence of infinitely many solutions; but instead of hypotheses (1.5) and (1.6) the nonlinearity $f$ is supposed to satisfy the assumption (1.3) above where $\Gamma(t)=|t|^{\sigma}$ with $\sigma>\max \left\{1, \frac{N}{p}\right\}$, and $F(x, t) \geq 0$ for any $(x,|t|) \in \Omega \times[R,+\infty)$ is such that $\lim _{|t| \rightarrow+\infty} \frac{F(x, t)}{|t|^{\phi^{0}}}=+\infty$. In the same paper, the authors obtained also similar existence result under the following assumption instead of (1.3): there exist $\mu>q$ and $\theta>0$ such that

$$
\mu F(x, t) \leq t f(x, t)+\theta|t|^{p}, \quad \forall(x, t) \in \Omega \times \mathbb{R} .
$$

Now, we give some review results concerning the case where the potential $V \not \equiv 0$ on $\Omega$. In [1], Abdou and Marcos, proved the existence of multiple solutions for a Dirichlet problem involving the $p(x)$-Laplacian operator with a changing sign potential $V$ belonging to a generalized Lebesgue space $L^{s(x)}(\Omega)$ when the nonlinearity $f$ satisfies some growth condition under (A-R) condition. In that work, the main assumptions on the variable exponents $q(\cdot), s(\cdot)$ and $p(\cdot)$ are such that: $q, s, p \in \mathcal{C}_{+}(\bar{\Omega})$ (see notation below) and satisfy $1<q(x)<p(x) \leq N<s(x)$ for any $x \in \bar{\Omega}$.

Recently, in 35 the authors proved the existence of nontrivial non-negative and non-positive solutions, and obtained infinitely many solutions for the quasilinear equation $-\operatorname{div} A(x, \nabla u)+$ $V(x)|u|^{\alpha(x)-2} u=f(x, u)$ in $\mathbb{R}^{N}$, where the divergence type operator has behaviors like $|\zeta|^{q(x)-2}$ for small $|\zeta|$ and like $|\zeta|^{p(x)-2}$ for large $|\zeta|$, where $1<\alpha(\cdot) \leq p(\cdot)<q(\cdot)<N$. In that paper, it is supposed that the potential $V \in L_{l o c}^{1}\left(\mathbb{R}^{N}\right)$ verifies $V(\cdot) \geq V_{0}>0, V(x) \rightarrow+\infty$ as $|x| \rightarrow+\infty$ and that the nonlinearity $f$ satisfies some growth condition with the following assumption instead of (A-R) condition: there exist constants $M, C_{1}, C_{2}>0$ and a function $a$ such that

$$
C_{1}|t|^{q(x)}[\ln (e+|t|)]^{a(x)-1} \leq C_{2} \frac{f(x, t) t}{\ln (e+|t|)} \leq f(x, t) t-s(x) F(x, t), \forall(x,|t|) \in \mathbb{R}^{N} \times[M,+\infty),
$$

where $\operatorname{ess}_{x \in \mathbb{R}^{N}} \inf (a(x)-q(x))>0, q(\cdot) \leq s(\cdot)$ and $\operatorname{ess}_{x \in \mathbb{R}^{N}} \inf \left(p^{*}(x)-s(x)\right)>0$ with $p^{*}(x):=$ $\frac{N p(x)}{N-p(x)}$. Related to this subject, we refer the readers to some important results concerning the study of the eigenvalue problems (see [3, 5, 6, 7, 8, 23, 24, 31] and the references therein). 
A main motivation of our current study is that, to the best of our knowledge, there is little research considering both the potential $V \not \equiv 0$ and nonlinearity $f$ without (A-R) condition for more general quasilinear equation in the framework of Musielak-Sobolev spaces. In this paper, our main goal is to show the existence of weak solutions to the problem $(P)$. Firstly, by using standard lower semicontinuity argument, we prove the existence of weak solutions under the condition that $V \in L^{s(x)}(\Omega)$ has changing sign, and the nonlinearity $f$ satisfies the condition $\left(f_{0}\right)$ below. Secondly, we establish the existence of at least a nontrivial solution and the existence of infinitely many solutions by using Mountain Pass Theorem and Fountain theorem respectively, where $V \in L^{s(x)}(\Omega)$ has constant sign and the nonlinearity $f$ does not satisfy the (A-R) condition. For these purposes, we propose a set of growth conditions under which we are able to check the Palais-Smale condition. More precisely, we prove the boundedness of Palais-Smale sequences by using a similar condition to that in (1.3) above instead of (A-R) condition.

The paper is organized as follows: In Section 2, we recall some definitions and basic properties about Musielak-Orlicz-Sobolev spaces and variable exponent Lebesgue-Sobolev spaces. In Section 3 , we state our main results and in Section 4 we give the proofs. Finally, in Section 5, we give an application of our main results.

\section{Preliminary Results}

In the study of nonlinear partial differential equations, it is well known that more general functional space can handle differential equations with more nonlinearities. For example, the $p$ Laplacian equations correspond to the classical Sobolev space setting, the $p(x)$-Laplacian equations correspond to the variable exponent Sobolev space setting, etc. Concerning the problem $(P)$, Musielak-Sobolev spaces are the adequate functional spaces corresponding to the solutions. We shall therefore start by recalling some basic facts about these spaces. For more details we refer the readers to the papers [22, 32, 15, 27.

Define

$$
\Phi(x, t)=\int_{0}^{t} \phi(x, s) s d s, \quad \forall t \geq 0 .
$$

Since the function $\phi$ satisfies the condition $(\phi)$, then $\Phi$ is a generalized $N$-function, that is, for each $t \in[0,+\infty), \Phi(., t)$ is measurable and for a.e. $x \in \Omega, \Phi(x,$.$) is continuous, even, convex,$ $\Phi(x, 0)=0, \Phi(x, t)>0$ for $t>0$ which satisfies the conditions

$$
\lim _{t \rightarrow 0^{+}} \frac{\Phi(x, t)}{t}=0 \text { and } \lim _{t \rightarrow+\infty} \frac{\Phi(x, t)}{t}=+\infty .
$$

We denote by $N(\Omega)$ the set of generalized $N$-functions. For $\Phi \in N(\Omega)$, the Musielak-Orlicz space $L^{\Phi}(\Omega)$ is defined by

$$
L^{\Phi}(\Omega):=\left\{u: u: \Omega \rightarrow \mathbb{R} \text { is measurable, and } \exists \lambda>0 \text { such that } \int_{\Omega} \Phi\left(x, \frac{|u(x)|}{\lambda}\right) d x<\infty\right\}
$$

endowed with the Luxemburg norm

$$
\|u\|_{L^{\Phi}(\Omega)}=\|u\|_{\Phi}:=\inf \left\{\lambda>0: \int_{\Omega} \Phi\left(x, \frac{|u(x)|}{\lambda}\right) d x \leq 1\right\} .
$$

Next, we define the Musielak-Sobolev space $W^{1, \Phi}(\Omega)$ by

$$
W^{1, \Phi}(\Omega):=\left\{u \in L^{\Phi}(\Omega):|\nabla u| \in L^{\Phi}(\Omega)\right\}
$$

endowed with the norm

$$
\|u\|_{W^{1, \Phi}(\Omega)}=\|u\|_{1, \Phi}:=\|u\|_{\Phi}+\|\nabla u\|_{\Phi},
$$

where $\|\nabla u\|_{\Phi}=\|\mid \nabla u\|_{\Phi}$. 
Remark 2.1. In the particular case where $\Phi(x, t)=\Phi(t)$ is independent of $x, W^{1, \Phi}(\Omega)$ is actually an Orlicz-Sobolev space while in the case where $\Phi(x, t)=|t|^{p(x)}$, this space becomes the variable exponent Sobolev space $W^{1, p(.)}(\Omega)$.

The function $\tilde{\Phi}: \Omega \times[0,+\infty) \rightarrow[0,+\infty)$ defined by

$$
\tilde{\Phi}(x, t)=\sup _{s>0}(t s-\Phi(x, s)), \quad \text { for } x \in \Omega \text { and } t \geq 0,
$$

is called the complementary function to $\Phi$ in the sense of Young. We observe that the function $\tilde{\Phi}$ belongs to $N(\Omega)$, and $\Phi$ is also the complementary function to $\tilde{\Phi}$. Furthermore, $\Phi$ and $\tilde{\Phi}$ satisfy the Young inequality

$$
s t \leq \Phi(x, t)+\tilde{\Phi}(x, s), \quad \text { for } x \in \Omega \text { and } s, t \geq 0 .
$$

Throughout this paper, we assume that there exist two positive constants $\phi_{0}$ and $\phi^{0}$ such that

$$
1<\phi_{0} \leq \frac{\phi(x, t) t^{2}}{\Phi(x, t)} \leq \phi^{0}<N, \quad \text { for } x \in \Omega \text { and } t>0
$$

This relation gives the following result (see [30, Proposition 2.1]):

Lemma 2.1. Let $u \in L^{\Phi}(\Omega)$ and $\rho, t \geq 0$, then we have

$$
\begin{gathered}
\min \left\{\rho^{\phi_{0}}, \rho^{\phi^{0}}\right\} \Phi(x, t) \leq \Phi(x, \rho t) \leq \max \left\{\rho^{\phi_{0}}, \rho^{\phi^{0}}\right\} \Phi(x, t), \\
\min \left\{\|u\|_{\Phi}^{\phi_{0}},\|u\|_{\Phi}^{\phi^{0}}\right\} \leq \int_{\Omega} \Phi(x,|u(x)|) d x \leq \max \left\{\|u\|_{\Phi}^{\phi_{0}},\|u\|_{\Phi}^{\phi^{0}}\right\} .
\end{gathered}
$$

Using the previous lemma we can easily show the following result:

Proposition 2.1. The function $\Phi$ satisfies the $\left(\Delta_{2}\right)$-condition, that is, there exist a positive constant $C>0$ such that

$$
\Phi(x, 2 t) \leq C \Phi(x, t), \quad \text { for } x \in \Omega \text { and } t \geq 0 .
$$

For the complementary function $\tilde{\Phi}$ we have the following analog lemma (see [19]).

Lemma 2.2. Let $u \in L^{\tilde{\Phi}}(\Omega)$ and $\rho, t \geq 0$, then we have

$$
\begin{gathered}
\min \left\{\rho^{\left(\phi_{0}\right)^{\prime}}, \rho^{\left(\phi^{0}\right)^{\prime}}\right\} \tilde{\Phi}(x, t) \leq \tilde{\Phi}(x, \rho t) \leq \max \left\{\rho^{\left(\phi_{0}\right)^{\prime}}, \rho^{\left(\phi^{0}\right)^{\prime}}\right\} \tilde{\Phi}(x, t), \\
\min \left\{\|u\|_{\tilde{\Phi}}^{\left(\phi_{0}\right)^{\prime}},\|u\|_{\tilde{\Phi}}^{\left(\phi^{0}\right)^{\prime}}\right\} \leq \int_{\Omega} \tilde{\Phi}(x,|u(x)|) d x \leq \max \left\{\|u\|_{\tilde{\Phi}}^{\left(\phi_{0}\right)^{\prime}},\|u\|_{\tilde{\Phi}}^{\left(\phi^{0}\right)^{\prime}}\right\},
\end{gathered}
$$

where $\left(\phi_{0}\right)^{\prime}=\frac{\phi_{0}}{\phi_{0}-1}$ and $\left(\phi^{0}\right)^{\prime}=\frac{\phi^{0}}{\phi^{0}-1}$.

Remark 2.2. From Lemma 2.2, the complementary function $\tilde{\Phi}$ also satisfies $\left(\Delta_{2}\right)$-condition.

Since both $\Phi$ and $\tilde{\Phi}$ satisfy the $\left(\Delta_{2}\right)$-condition, then we have the following result:

Proposition 2.2 (27]). The following assertions hold:

(1) $L^{\Phi}(\Omega)=\left\{u: u: \Omega \rightarrow \mathbb{R}\right.$ is measurable, and $\left.\int_{\Omega} \Phi(x,|u(x)|) d x<\infty\right\}$

(2) For any sequence $\left(u_{n}\right)$ in $L^{\Phi}(\Omega)$, we have

a) $\int_{\Omega} \Phi\left(x,\left|u_{n}(x)\right|\right) d x \rightarrow 0($ resp. $1 ;+\infty) \Leftrightarrow\left\|u_{n}\right\|_{\Phi} \rightarrow 0($ resp. $1 ;+\infty)$,

b) $u_{n} \rightarrow u$ in $L^{\Phi}(\Omega) \Rightarrow \int_{\Omega}\left|\Phi\left(x,\left|u_{n}(x)\right|\right)-\Phi(x,|u(x)|)\right| d x \rightarrow 0$ as $n \rightarrow+\infty$.

(3) Let $u \in L^{\Phi}(\Omega)$ and $v \in L^{\tilde{\Phi}}(\Omega)$. Then the Hölder type inequality holds true

$$
\left|\int_{\Omega} u(x) v(x) d x\right| \leq 2\|u\|_{\Phi}\|v\|_{\tilde{\Phi}}
$$

(4) $\phi(x,|u(x)|) u(x) \in L^{\tilde{\Phi}}(\Omega)$ provided that $u \in L^{\Phi}(\Omega)$. 
Let $\Phi, \Psi \in N(\Omega)$. We say that $\Phi$ is weaker than $\Psi$, and denote $\Phi \preccurlyeq \Psi$, if there exist positive constants $K_{1}, K_{2}$ and a nonnegative function $h \in L^{1}(\Omega)$ such that

$$
\Phi(x, t) \leq K_{1} \Psi\left(x, K_{2} t\right)+h(x), \quad \text { for } x \in \Omega \text { and } t \geq 0 .
$$

By Theorem 8.5 in 32 , the following embeddings

$$
L^{\Psi}(\Omega) \hookrightarrow L^{\Phi}(\Omega) \text { and } L^{\tilde{\Phi}}(\Omega) \hookrightarrow L^{\tilde{\Psi}}(\Omega)
$$

are continuous provided that $\Phi, \Psi$ are such that $\Phi \preccurlyeq \Psi$.

We say that $\Phi \in N(\Omega)$ is locally integrable if $\Phi\left(., t_{0}\right) \in L^{1}(\Omega)$ for every $t_{0}>0$. We point out that when $\Phi$ is locally integrable, then $\left(L^{\Phi}(\Omega),\|\cdot\|_{\Phi}\right)$ is a separable Banach space (see [22, 32]).

In this paper, we shall need the following assumptions.

$\left(\phi_{1}\right) \inf _{x \in \Omega} \Phi(x, 1)=c_{1}>0$.

$\left(\phi_{2}\right)$ For every $t_{0}>0$ there exists $c=c\left(t_{0}\right)>0$ such that

$$
\frac{\Phi(x, t)}{t} \geq c, \quad \text { and } \quad \frac{\tilde{\Phi}(x, t)}{t} \geq c \text { for } x \in \Omega \text { and } t \geq t_{0} .
$$

We note that, $\left(\phi_{2}\right) \Rightarrow\left(\phi_{1}\right)$. Moreover, in the case where $\Phi$ is independent of $x,\left(\phi_{1}\right)$ and $\left(\phi_{2}\right)$ hold automatically and $\Phi$ is automatically locally integrable.

By assumption $\left(\phi_{1}\right)$, we have the following embeddings

$$
L^{\Phi}(\Omega) \hookrightarrow L^{1}(\Omega) \quad \text { and } \quad W^{1, \Phi}(\Omega) \hookrightarrow W^{1,1}(\Omega) .
$$

In addition, by assuming $\Phi$ and $\tilde{\Phi}$ both locally integrable and satisfy $\left(\phi_{2}\right)$, we conclude that $L^{\Phi}(\Omega)$ is reflexive, and that the mapping $J: L^{\tilde{\Phi}}(\Omega) \rightarrow\left(L^{\Phi}(\Omega)\right)^{*}$ defined by

$$
\langle J(v), w\rangle=\int_{\Omega} v(x) w(x) d x, \quad \forall v \in L^{\tilde{\Phi}}(\Omega), \forall w \in L^{\Phi}(\Omega)
$$

is a linear isomorphism and $\|J(v)\|_{\left(L^{\Phi}(\Omega)\right)^{*}} \leq 2\|v\|_{L^{\tilde{\Phi}}(\Omega)}$ (see [32, p. 189].)

We denote by $W_{0}^{1, \Phi}(\Omega)$ the closure of $\mathcal{C}_{0}^{\infty}(\Omega)$ in $W^{1, \Phi}(\Omega)$ and by $\mathcal{D}_{0}^{1, \Phi}(\Omega)$ the completion of $\mathcal{C}_{0}^{\infty}(\Omega)$ in the norm $\|\nabla u\|_{\Phi}$. It is clear that $W_{0}^{1, \Phi}(\Omega)=\mathcal{D}_{0}^{1, \Phi}(\Omega)$ in the case where $\|\nabla u\|_{\Phi}$ is an equivalent norm in $W_{0}^{1, \Phi}(\Omega)$.

By assuming $\Phi$ locally integrable and satisfies $\left(\phi_{1}\right), W^{1, \Phi}(\Omega), W_{0}^{1, \Phi}(\Omega)$ and $\mathcal{D}_{0}^{1, \Phi}(\Omega)$ are clearly separable Banach spaces, and we have

$$
\begin{aligned}
& W_{0}^{1, \Phi}(\Omega) \hookrightarrow W^{1, \Phi}(\Omega) \hookrightarrow W^{1,1}(\Omega) \\
& \mathcal{D}_{0}^{1, \Phi}(\Omega) \hookrightarrow \mathcal{D}_{0}^{1,1}(\Omega)=W_{0}^{1,1}(\Omega) .
\end{aligned}
$$

In addition, these spaces are reflexive if $L^{\Phi}(\Omega)$ is reflexive.

In this work, we need to use some standard tools such as the Poincaré inequality and results of compactness for embeddings in Musielak-Sobolev spaces. For this reason, we shall suppose the following supplementary assumptions on $\Phi$.

$\left(H_{1}\right) \Omega \subset \mathbb{R}^{N}(N \geq 2)$ is a bounded domain with the cone property, $\Phi \in N(\Omega)$.

$\left(H_{2}\right) \Phi: \bar{\Omega} \times[0,+\infty) \rightarrow[0,+\infty)$ is continuous and $\Phi(x, t) \in(0,+\infty)$ for $x \in \bar{\Omega}$ and $t \in(0,+\infty)$. Now, let $\Phi$ satisfies $\left(H_{1}\right)$ and $\left(H_{2}\right)$. Then, for each $x \in \bar{\Omega}$, the function $\Phi(x, \cdot):[0,+\infty) \rightarrow[0,+\infty)$ is a strictly increasing homeomorphism. Denote by $\Phi^{-1}(x, \cdot)$ the inverse function of $\Phi(x, \cdot)$. We also assume the following condition.

$\left(H_{3}\right)$

$$
\int_{0}^{1} \frac{\Phi^{-1}(x, t)}{t^{\frac{N+1}{N}}} d t<+\infty, \forall x \in \bar{\Omega}
$$


Define the function $\Phi_{*}^{-1}: \bar{\Omega} \times[0,+\infty) \rightarrow[0,+\infty)$ by

$$
\Phi_{*}^{-1}(x, s)=\int_{0}^{s} \frac{\Phi^{-1}(x, \tau)}{\tau^{\frac{N+1}{N}}} d \tau, \text { for } x \in \bar{\Omega} \text { and } s \in[0,+\infty) .
$$

Then, by assumption $\left(H_{3}\right), \Phi_{*}^{-1}$ is well defined, and for each $x \in \bar{\Omega}, \Phi_{*}^{-1}(x, \cdot)$ is strictly increasing, $\Phi_{*}^{-1}(x, \cdot) \in \mathcal{C}^{1}((0,+\infty))$ and the function $\Phi_{*}^{-1}(x, \cdot)$ is concave.

Set $T(x)=\lim _{s \rightarrow+\infty} \Phi_{*}^{-1}(x, s)$, for all $x \in \bar{\Omega}$. Then, $T(x) \in(0,+\infty]$. Define the function $\Phi_{*}:$ $\bar{\Omega} \times[0,+\infty) \rightarrow[0,+\infty)$ by

$$
\Phi_{*}(x, t)= \begin{cases}s, & \text { if } x \in \bar{\Omega}, t \in[0, T(x)) \text { and } \Phi_{*}^{-1}(x, s)=t \\ +\infty, & \text { if } x \in \bar{\Omega}, t \geq T(x) .\end{cases}
$$

Then $\Phi_{*} \in N(\Omega)$, and for each $x \in \bar{\Omega}, \Phi_{*}(x, \cdot) \in \mathcal{C}^{1}((0, T(x)))$. $\Phi_{*}$ is called the Sobolev conjugate function of $\Phi$.

Let $X$ be a metric space and $f: X \rightarrow(-\infty,+\infty]$ be an extended real-valued function. For $x \in X$ with $f(x) \in \mathbb{R}$, the continuity of $f$ at $x$ is well defined. Now, for $x \in X$ with $f(x)=+\infty$, we say that $f$ is continuous at $x$ if given any $M>0$, there exists a neighborhood $U$ of $x$ such that $f(y)>M$ for all $y \in U$. We say that $f: X \rightarrow(-\infty,+\infty]$ is continuous on $X$ if $f$ is continuous at every $x \in X$. Define $\operatorname{Dom}(f)=\{x \in X: f(x) \in \mathbb{R}\}$ and denote by $\mathcal{C}^{1-0}(X)$ the set of all locally Lipschitz continuous real-valued functions defined on $X$.

Remark 2.3. Suppose that $\Phi$ satisfies $\left(H_{2}\right)$. Then, for each $t_{0} \geq 0, \tilde{\Phi}\left(x, t_{0}\right)$ and $\Phi_{*}\left(x, t_{0}\right)$ are bounded.

Concerning the function $\Phi_{*}$ and the operator $T$, we suppose that

$\left(H_{4}\right) T: \bar{\Omega} \rightarrow[0,+\infty]$ is continuous on $\bar{\Omega}$ and $T \in \mathcal{C}^{1-0}(\operatorname{Dom}(T))$;

$\left(H_{5}\right) \Phi_{*} \in \mathcal{C}^{1-0}\left(\operatorname{Dom}\left(\Phi_{*}\right)\right)$ and there exist positive constants $C_{0}, \delta_{0}<\frac{1}{N}$ and $t_{0} \in\left(0, \min _{x \in \bar{\Omega}} T(x)\right)$ such that

$$
\left|\nabla_{x} \Phi_{*}(x, t)\right| \leq C_{0}\left(\Phi_{*}(x, t)\right)^{1+\delta_{0}},
$$

for $x \in \Omega$ and $t \in\left[t_{0}, T(x)\right)$ provided that $\nabla_{x} \Phi_{*}(x, t)$ exists.

Remark 2.4. Examples of generalized $N$-function $\Phi$ satifying the above assumptions and covering the case of variable exponent space, double-phase space, and variable exponent double-phase space, are given in [36].

Let $\Phi, \Psi \in N(\Omega)$. We say that $\Phi$ essentially grows more slowly that $\Psi$ and we write $\Phi \ll \Psi$, if for any $k>0$,

$$
\lim _{t \rightarrow+\infty} \frac{\Phi(x, k t)}{\Psi(x, t)}=0 \text { uniformly for } x \in \Omega .
$$

Obviously, if $\Phi \ll \Psi$ then $\Phi \preccurlyeq \Psi$.

Now, we recall the following embedding theorems for Musielak-Sobolev spaces (see [15, 27]).

Theorem 2.1. Assume $\left(H_{1}\right)-\left(H_{5}\right)$ hold. Then

(1) There is a continuous embedding $W^{1, \Phi}(\Omega) \hookrightarrow L^{\Phi_{*}}(\Omega)$.

(2) Suppose that $\Psi \in N(\Omega), \Psi: \bar{\Omega} \times[0,+\infty) \rightarrow[0,+\infty)$ is continuous, and $\Psi(x, t) \in(0,+\infty)$ for $x \in \Omega$ and $t \in(0,+\infty)$. If $\Psi \ll \Phi_{*}$. Then, the embedding $W^{1, \Phi}(\Omega) \hookrightarrow \hookrightarrow L^{\Psi}(\Omega)$ is compact.

In particular, as $\Phi \ll \Phi_{*}$ then we have

Theorem 2.2. Assume $\left(H_{1}\right)-\left(H_{5}\right)$ hold. Then

(1) The embedding $W^{1, \Phi}(\Omega) \hookrightarrow \hookrightarrow L^{\Phi}(\Omega)$ is compact . 
(2) The Poincaré type inequality

$$
\|u\|_{\Phi} \leq C\|\nabla u\|_{\Phi} \quad \text { for } u \in W_{0}^{1, \Phi}(\Omega),
$$

holds.

We finish the recall of Musielak-Sobolev spaces properties by giving the following analog lemma (see [19]).

Lemma 2.3. Let $u \in L^{\Phi_{*}}(\Omega)$ and $\rho, t \geq 0$. Then, we have

$$
\begin{gathered}
\min \left\{\rho^{\left(\phi_{0}\right)^{*}}, \rho^{\left(\phi^{0}\right)^{*}}\right\} \Phi_{*}(x, t) \leq \Phi_{*}(x, \rho t) \leq \max \left\{\rho^{\left(\phi_{0}\right)^{*}}, \rho^{\left(\phi^{0}\right)^{*}}\right\} \Phi_{*}(x, t), \\
\min \left\{\|u\|_{\Phi_{*}}^{\left(\phi_{0}\right)^{*}},\|u\|_{\Phi_{*}}^{\left(\phi^{0}\right)^{*}}\right\} \leq \int_{\Omega} \Phi_{*}(x,|u(x)|) d x \leq \max \left\{\|u\|_{\Phi_{*}}^{\left(\phi_{0}\right)^{*}},\|u\|_{\Phi_{*}}^{\left(\phi^{0}\right)^{*}}\right\},
\end{gathered}
$$

where $\left(\phi_{0}\right)^{*}=\frac{N \phi_{0}}{N-\phi_{0}}$ and $\left(\phi^{0}\right)^{*}=\frac{N \phi^{0}}{N-\phi^{0}}$.

Now, we give some background facts concerning the variable exponent Lebesgue spaces. For more details on the basic properties of these spaces, we refer the reader to the papers [18, 25].

Set

$$
\mathcal{C}_{+}(\bar{\Omega})=\{h \in \mathcal{C}(\bar{\Omega}): h(x)>1 \text { for any } x \in \bar{\Omega}\} .
$$

For any $h \in \mathcal{C}_{+}(\bar{\Omega})$ we define:

$$
h^{-}=\min _{x \in \bar{\Omega}} h(x), \quad h^{+}=\max _{x \in \bar{\Omega}} h(x) .
$$

For any $q(x) \in \mathcal{C}_{+}(\bar{\Omega})$, we define the variable exponent Lebesgue space:

$$
L^{q(x)}(\Omega):=\left\{u: u: \Omega \rightarrow \mathbb{R} \text { is measurable with } \int_{\Omega}|u(x)|^{q(x)} d x<\infty\right\},
$$

endowed with the norm

$$
\|u\|_{L^{q(x)}(\Omega)}=\|u\|_{q(x)}:=\inf \left\{\lambda>0: \int_{\Omega}\left|\frac{u(x)}{\lambda}\right|^{q(x)} d x \leq 1\right\} .
$$

We recall that the variable exponent Lebesgue spaces are separable and reflexive Banach spaces. Let $L^{q^{\prime}(x)}(\Omega)$ denote the conjugate space of $L^{q(x)}(\Omega)$ with $\frac{1}{q(x)}+\frac{1}{q^{\prime}(x)}=1$. For any $u \in L^{q(x)}(\Omega)$ and $v \in L^{q^{\prime}(x)}(\Omega)$, the Hölder type inequality

$$
\int_{\Omega}|u v| d x \leq\left(\frac{1}{q^{-}}+\frac{1}{q^{\prime-}}\right)\|u\|_{q(x)}\|v\|_{q^{\prime}(x)},
$$

holds true. Moreover, if $0<|\Omega|<\infty$ and $q_{1}, q_{2}$ are variable exponents so that $q_{1}(x) \leq q_{2}(x)$ almost everywhere in $\Omega$, then there exists the continuous embedding $L^{q_{2}(x)}(\Omega) \hookrightarrow L^{q_{1}(x)}(\Omega)$. Furthermore, if we define the mapping $\rho_{q}: L^{q(x)}(\Omega) \rightarrow \mathbb{R}^{+}$by

$$
\rho_{q}(u)=\int_{\Omega}|u|^{q(x)} d x
$$

then the following relations hold true:

$$
\begin{gathered}
\min \left\{\|u\|_{q(x)}^{q_{-}},\|u\|_{q(x)}^{q_{+}}\right\} \leq \rho_{q}(u) \leq \max \left\{\|u\|_{q(x)}^{q_{-}},\|u\|_{q(x)}^{q_{+}}\right\}, \\
\|u\|_{q(x)}<1(=1,>1) \Leftrightarrow \rho_{q}(u)<1(=1,>1), \\
\left\|u_{n}-u\right\|_{q(x)} \rightarrow 0 \Leftrightarrow \rho_{q}\left(u_{n}-u\right) \rightarrow 0, \quad \forall\left(u_{n}\right), u \in L^{q(x)}(\Omega), \\
\left\|u_{n}\right\|_{q(x)} \rightarrow+\infty \Leftrightarrow \rho_{q}\left(u_{n}\right) \rightarrow+\infty, \quad \forall\left(u_{n}\right) \in L^{q(x)}(\Omega) .
\end{gathered}
$$

We recall also the following proposition, which will be used later: 
Proposition 2.3 ([14]). Let $p$ and $q$ be measurable functions such that $p \in L^{\infty}(\Omega)$ and $1<$ $p(x) q(x) \leq \infty$ for a.e. $x \in \Omega$. Let $u \in L^{q(x)}(\Omega), u \neq 0$. Then

$$
\begin{aligned}
& \|u\|_{p(x) q(x)} \leq 1 \Rightarrow\|u\|_{p(x) q(x)}^{p^{+}} \leq\left\||u|^{p(x)}\right\|_{q(x)} \leq\|u\|_{p(x) q(x)}^{p^{-}}, \\
& \|u\|_{p(x) q(x)} \geq 1 \Rightarrow\|u\|_{p(x) q(x)}^{p^{-}} \leq\left\||u|^{p(x)}\right\|_{q(x)} \leq\|u\|_{p(x) q(x)}^{p^{+}} .
\end{aligned}
$$

In particular when $p(x)=p$ is a constant, then

$$
\left\||u|^{p}\right\|_{q(x)}=\|u\|_{p q(x)}^{p} \text {. }
$$

\section{MAIN RESUlts}

In this section we state the main results of this paper. We will study the problem $(P)$ when $q \in \mathcal{C}_{+}(\bar{\Omega})$ and the potential $V: \Omega \rightarrow \mathbb{R}$ is nontrivial and belongs to $L^{s(x)}(\Omega)$ with $s \in \mathcal{C}(\bar{\Omega})$. Before dealing with our main results in this section, we introduce the following assumptions for $f(x, u)$ :

$\left(f_{0}\right)$ There exists $\Psi \in N(\Omega)$ satisfying the assumption (2) of Theorem 2.1, and two positive constants $\psi_{0}$ and $\psi^{0}$ such that

$$
\begin{gathered}
1<\psi_{0} \leq \frac{\psi(x, t) t}{\Psi(x, t)} \leq \psi^{0}, \text { for } x \in \Omega \text { and } t>0 . \\
|f(x, t)| \leq C_{1} \psi(x,|t|)+h(x), \text { for }(x, t) \in \Omega \times \mathbb{R},
\end{gathered}
$$

where $C_{1}$ is a positive constant, $0 \leq h \in L^{\tilde{\Psi}}(\Omega)$, and $\psi: \Omega \times \mathbb{R}^{+} \rightarrow \mathbb{R}^{+}$is a continuous function and $\Psi(x, t)=\int_{0}^{t} \psi(x, s) d s$, for all $x \in \Omega$.

$\left(f_{1}\right)$ There exists $\Gamma \in N(\Omega)$ satisfying the assumptions of $\left(H_{2}\right)$, and two positive constants $\gamma_{0}$ and $\gamma^{0}$ such that

$$
\begin{gathered}
1<\frac{N}{\phi_{0}}<\gamma_{0} \leq \frac{\gamma(x, t) t}{\Gamma(x, t)} \leq \gamma^{0}, \text { for } x \in \Omega \text { and } t>0 . \\
\Gamma\left(x, \frac{F(x, t)}{|t| \phi_{0}}\right) \leq C_{2} H(x, t), \text { for } x \in \Omega \text { and }|t| \geq M,
\end{gathered}
$$

where $C_{2}, M$ are positive constants, $H(x, t)=f(x, t) t-\nu F(x, t)$, for all $(x, t) \in \Omega \times \mathbb{R}$ with $\nu=\phi^{0}$ if $V \leq 0$ a.e. on $\Omega$ and $\nu=q^{+}$if $V \geq 0$ a.e. on $\Omega$, and $\gamma: \Omega \times \mathbb{R}^{+} \rightarrow \mathbb{R}^{+}$is a continuous function and $\Gamma(x, t)=\int_{0}^{t} \gamma(x, s) d s$, for all $x \in \Omega$.

$\left(f_{2}\right) \lim _{|t| \rightarrow+\infty} \frac{F(x, t)}{|t|^{\phi^{0}}}=+\infty$, uniformly for $x \in \Omega$.

$\left(f_{3}\right) f(x, t)=o(|t| \phi(x, t))$ as $t \rightarrow 0$, uniformly for $x \in \Omega$.

$\left(f_{4}\right) f(x,-t)=-f(x, t)$ for all $(x, t) \in \Omega \times \mathbb{R}$.

To summarize all assumptions concerning the function $\Phi$, in what follows we shall say that the function $\Phi$ satisfies the assumption ( $\Phi)$ if: $\phi$ satisfies the assumption $(\phi), \Phi$ satisfies (2.1) and $\left(H_{1}\right)-\left(H_{5}\right)$, both $\Phi$ and $\tilde{\Phi}$ are locally integrable and satisfy $\left(\phi_{2}\right)$. Hence, under the assumption $(\Phi)$, the spaces $L^{\Phi}(\Omega), W^{1, \Phi}(\Omega), W_{0}^{1, \Phi}(\Omega)$ are separable reflexive Banach spaces. Therefore, we can apply the embedding theorems for Musielak-Sobolev spaces in Theorem 2.1] and Theorem 2.2

Definition 3.1. A function $u \in W_{0}^{1, \Phi}(\Omega)$ is said to be a weak solution of problem $(P)$ if it holds that

$$
\int_{\Omega} \phi(x,|\nabla u|) \nabla u \nabla v d x+\int_{\Omega} V(x)|u|^{q(x)-2} u v d x=\int_{\Omega} f(x, u) v d x, \quad \forall v \in W_{0}^{1, \Phi}(\Omega) .
$$

Our main results in this paper are given by the following theorems: 
Theorem 3.1. Assume that the assumptions $(\Phi)$ and $\left(f_{0}\right)$ hold. Furthermore, assume that $\max \left\{\psi^{0}, q^{+}\right\}<\phi_{0}$ and $s(x)>\frac{q(x)\left(\phi_{0}\right)^{*}}{\left(\phi_{0}\right)^{*}-q(x)}$ for every $x \in \bar{\Omega}$. Then, problem $(P)$ has a weak solution.

In order to obtain the second main result, we assume that $f$ satisfies the following condition $\left(f_{0}^{\prime}\right)$ instead of $\left(f_{0}\right)$ :

$\left(f_{0}^{\prime}\right)$ We assume that (3.1) of $\left(f_{0}\right)$ holds and that

$$
|f(x, t)| \leq C_{1}(\psi(x,|t|)+1), \text { for }(x, t) \in \Omega \times \mathbb{R},
$$

where $C_{1}$ is a positive constant.

Theorem 3.2. Assume that the assumptions $(\Phi)$ and $\left(f_{0}^{\prime}\right)-\left(f_{3}\right)$ hold. Furthermore, assume that $\phi^{0}<\min \left\{\psi_{0}, q^{-}\right\}, \max \left\{\psi^{0}, q^{+}\right\}<\left(\phi_{0}\right)^{*}, q^{+}-\frac{1}{2} \phi_{0}<q^{-}$and $s(x)>\frac{q(x)\left(\phi_{0}\right)^{*}}{\left(\phi_{0}\right)^{*}-q(x)}$ for every $x \in \bar{\Omega}$. If $V$ has a constant sign a.e. on $\Omega$, then the problem $(P)$ has a nontrivial weak solution.

Theorem 3.3. Assume that the assumptions of Theorem 3.2 hold. If the function $f$ satisfies $\left(f_{4}\right)$, then the problem $(P)$ has a sequence of weak solutions $\left( \pm u_{n}\right)_{n \in \mathbb{N}} \subseteq W_{0}^{1, \Phi}(\Omega)$ such that $\mathcal{I}\left( \pm u_{n}\right) \rightarrow+\infty$ as $n \rightarrow+\infty$.

In order to prove Theorem 3.3 we will use the following Fountain theorem (see [37] for details). Let $(X,\|\cdot\|)$ be a real reflexive Banach space such that $X=\overline{\oplus_{j \in \mathbb{N}^{*}} X_{j}}$ with $\operatorname{dim}\left(X_{j}\right)<+\infty$ for any $j \in \mathbb{N}^{*}$. For each $k \in \mathbb{N}^{*}$, we set $Y_{k}=\oplus_{j=1}^{k} X_{j}$ and $Z_{k}=\overline{\oplus_{j=k}^{\infty} X_{j}}$.

Proposition 3.1 (Fountain theorem). Let $(X,\|\cdot\|)$ be a real reflexive Banach space and $\mathcal{I} \in$ $\mathcal{C}^{1}(X, \mathbb{R})$ an even functional. If for each sufficiently large $k \in \mathbb{N}^{*}$, there exist $\rho_{k}>r_{k}>0$ such that the following conditions hold:

(1) $\inf _{\left\{u \in Z_{k},\|u\|=r_{k}\right\}} \mathcal{I}(u) \rightarrow+\infty$ as $k \rightarrow+\infty$,

(2) $\max _{\left\{u \in Y_{k},\|u\|=\rho_{k}\right\}} \mathcal{I}(u) \leq 0$,

(3) I satisfies the Palais-Smale condition for every $c>0$,

then $\mathcal{I}$ has a sequence of critical values tending to $+\infty$.

\section{Proofs of the main Results}

In this section we give the proofs of our main results. We note that in these results we always have $s(x)>\frac{q(x)\left(\phi_{0}\right)^{*}}{\left(\phi_{0}\right)^{*}-q(x)}$ for every $x \in \bar{\Omega}$ and $\max \left\{\psi^{0}, q^{+}\right\}<\left(\phi_{0}\right)^{*}$.

Define the functional $\mathcal{I}: W_{0}^{1, \Phi}(\Omega) \rightarrow \mathbb{R}$ by the formula

$$
\mathcal{I}(u)=\mathcal{H}(u)+\mathcal{J}(u)-\mathcal{F}(u),
$$

where,

$$
\mathcal{H}(u)=\int_{\Omega} \Phi(x,|\nabla u|) d x, \quad \mathcal{J}(u)=\int_{\Omega} \frac{V(x)}{q(x)}|u|^{q(x)} d x, \quad \text { and } \quad \mathcal{F}(u)=\int_{\Omega} F(x, u) d x,
$$

with $F(x, t)=\int_{0}^{t} f(x, s) d s$.

Proposition 4.1. The functional $\mathcal{I}$ is well defined and $\mathcal{I} \in \mathcal{C}^{1}\left(W_{0}^{1, \Phi}(\Omega), \mathbb{R}\right)$ with the derivative given by

$$
\left\langle\mathcal{I}^{\prime}(u), v\right\rangle=\int_{\Omega} \phi(x,|\nabla u|) \nabla u \nabla v d x+\int_{\Omega} V(x)|u|^{q(x)-2} u v d x-\int_{\Omega} f(x, u) v d x, \quad \forall u, v \in W_{0}^{1, \Phi}(\Omega) .
$$


Proof. Firstly, it is clear that $\mathcal{H}$ is well defined on $W_{0}^{1, \Phi}(\Omega)$. Furthermore, by similar arguments used in the proof of [30, Lemma 4.2], we have $\mathcal{H} \in \mathcal{C}^{1}\left(W_{0}^{1, \Phi}(\Omega), \mathbb{R}\right)$ and its derivative is given by

$$
\left\langle\mathcal{H}^{\prime}(u), v\right\rangle=\int_{\Omega} \phi(x,|\nabla u|) \nabla u \nabla v d x, \quad \forall u, v \in W_{0}^{1, \Phi}(\Omega) .
$$

Secondly, the functional $\mathcal{J}$ is well defined. Indeed, since $s(x)>\frac{q(x)\left(\phi_{0}\right)^{*}}{\left(\phi_{0}\right)^{*}-q(x)}$ for every $x \in \bar{\Omega}$, then it is clear that $s \in \mathcal{C}_{+}(\bar{\Omega})$ and $s(x)>q(x)$ for every $x \in \bar{\Omega}$. Furthermore, by a simple computation we have,

$$
1<s^{\prime}(x) q(x)<\left(\phi_{0}\right)^{*} \quad \text { and } \quad 1<\alpha(x):=\frac{s(x) q(x)}{s(x)-q(x)}<\left(\phi_{0}\right)^{*}, \quad \forall x \in \bar{\Omega} .
$$

Thus,

$$
\max _{x \in \bar{\Omega}} s^{\prime}(x) q(x):=s^{\prime}\left(x_{0}\right) q\left(x_{0}\right)<\left(\phi_{0}\right)^{*} \text { and } \max _{x \in \bar{\Omega}} \alpha(x):=\alpha\left(x_{0}\right)<\left(\phi_{0}\right)^{*} .
$$

Using Lemma 2.3 and $\left(H_{5}\right)$, we obtain

$$
\lim _{t \rightarrow+\infty} \frac{|k t|^{s^{\prime}(x) q(x)}}{\Phi_{*}(x, t)} \leq \frac{k^{s^{\prime}\left(x_{0}\right) q\left(x_{0}\right)}}{\Phi_{*}(x, 1)} \lim _{t \rightarrow+\infty} \frac{1}{t^{\left(\phi_{0}\right)^{*}-s^{\prime}\left(x_{0}\right) q\left(x_{0}\right)}}=0 \text { uniformly for } x \in \Omega .
$$

Using the same arguments above we show that

$$
\lim _{t \rightarrow+\infty} \frac{|k t|^{\alpha(x)}}{\Phi_{*}(x, t)}=0 \text { uniformly for } x \in \Omega .
$$

Hence, (4.3) and (4.4) imply that $|t|^{s^{\prime}(x) q(x)} \ll \Phi_{*}$ and $|t|^{\alpha(x)} \ll \Phi_{*}$ respectively. Thus, from Theorem 2.1 we have the following compact embeddings

$$
W_{0}^{1, \Phi}(\Omega) \hookrightarrow \hookrightarrow L^{s^{\prime}(x) q(x)}(\Omega),
$$

and

$$
W_{0}^{1, \Phi}(\Omega) \hookrightarrow \hookrightarrow L^{\alpha(x)}(\Omega) .
$$

Now, by using the Hölder inequality, Proposition 2.3, and (4.5), we have for all $u$ in $W_{0}^{1, \Phi}(\Omega)$

$$
\begin{aligned}
|\mathcal{J}(u)| \leq c_{0}\|V\|_{s(x)}\left\||u|^{q(x)}\right\|_{s^{\prime}(x)} & \leq c_{1}\|V\|_{s(x)} \max \left\{\|u\|_{s^{\prime}(x) q(x)}^{q_{-}},\|u\|_{s^{\prime}(x) q(x)}^{q_{+}}\right\} \\
& \leq c_{2}\|V\|_{s(x)} \max \left\{\|u\|_{1, \Phi}^{q_{-}},\|u\|_{1, \Phi}^{q_{+}}\right\}
\end{aligned}
$$

where $c_{i}, i=0,1,2$ are positive constants. Hence, $\mathcal{J}$ is well defined. Moreover, since $q^{+}<\left(\phi_{0}\right)^{*}$ then, as in the proof of relation (4.3), the space $W_{0}^{1, \Phi}(\Omega)$ is compactly embedded in $L^{q(x)}(\Omega)$. Hence, using (4.6) and following the same arguments as in the proof of [23, Proposition 2], we obtain $\mathcal{J} \in \mathcal{C}^{1}\left(W_{0}^{1, \Phi}(\Omega), \mathbb{R}\right)$ and

$$
\left\langle\mathcal{J}^{\prime}(u), v\right\rangle=\int_{\Omega} V(x)|u|^{q(x)-2} u v d x, \quad \forall u, v \in W_{0}^{1, \Phi}(\Omega) .
$$

Finally, from the properties of $\Psi, \Psi(x, k)$ is bounded for any positive constant $k$. Using Lemma 2.1 and the fact that $\psi^{0}<\left(\phi_{0}\right)^{*}$ we obtain for any $k>0$

$$
\lim _{t \rightarrow+\infty} \frac{\Psi(x, k t)}{\Phi_{*}(x, t)} \leq \frac{\Psi(x, k)}{\Phi_{*}(x, 1)} \lim _{t \rightarrow+\infty} \frac{1}{t^{\left(\phi_{0}\right)^{*}-\psi^{0}}}=0 \text { uniformly for } x \in \Omega .
$$

Hence, $\Psi \ll \Phi_{*}$, which implies by Theorem 2.1 that

$$
W_{0}^{1, \Phi}(\Omega) \hookrightarrow \hookrightarrow L^{\Psi}(\Omega) .
$$


Consequently, from (3.2), the functional $\mathcal{F}$ is well defined and $\mathcal{F} \in \mathcal{C}^{1}\left(W_{0}^{1, \Phi}(\Omega), \mathbb{R}\right)$ with its derivative given by

$$
\left\langle\mathcal{F}^{\prime}(u), v\right\rangle=\int_{\Omega} f(x, u) v d x, \quad \forall u, v \in W_{0}^{1, \Phi}(\Omega) .
$$

The proof of this proposition is now complete.

\section{Proposition 4.2.}

i) The mapping $\mathcal{H}^{\prime}: W_{0}^{1, \Phi}(\Omega) \rightarrow\left(W_{0}^{1, \Phi}(\Omega)\right)^{*}$ defined by

$$
\left\langle\mathcal{H}^{\prime}(u), v\right\rangle=\int_{\Omega} \phi(x,|\nabla u|) \nabla u \nabla v d x, \quad \forall u, v \in W_{0}^{1, \Phi}(\Omega),
$$

is bounded, coercive, strictly monotone homeomorphism, and is of type $\left(S_{+}\right)$, namely,

$$
u_{n} \rightarrow u \text { in } W_{0}^{1, \Phi}(\Omega) \text { and } \limsup _{n \rightarrow \infty}\left\langle\mathcal{H}^{\prime}\left(u_{n}\right), u_{n}-u\right\rangle \leq 0 \text { imply that } u_{n} \rightarrow u \text { in } W_{0}^{1, \Phi}(\Omega) \text {, }
$$

where $\rightarrow$ and $\rightarrow$ denote the weak and strong convergence in $W_{0}^{1, \Phi}(\Omega)$, respectively.

ii) The functional $\mathcal{F}$ is sequentially weakly continuous, namely, $u_{n} \rightarrow u$ in $W_{0}^{1, \Phi}(\Omega)$ implies $\mathcal{F}\left(u_{n}\right) \rightarrow \mathcal{F}(u)$. In addition, the mapping $\mathcal{F}^{\prime}: W_{0}^{1, \Phi}(\Omega) \rightarrow\left(W_{0}^{1, \Phi}(\Omega)\right)^{*}$ defined by

$$
\left\langle\mathcal{F}^{\prime}(u), v\right\rangle=\int_{\Omega} f(x, u) v d x, \quad \forall u, v \in W_{0}^{1, \Phi}(\Omega),
$$

is a completely continuous linear operator.

Proof. We refer the reader to [16, Theorem 2.2] for the proof of the first item and to [27, Lemma 4.1] for that of the second one.

We note that, by Proposition 4.1 and Definition $3.1 u$ is a weak solution of problem $(P)$ if and only if $u$ is a critical point of the functional $\mathcal{I}$. Hence, we shall use critical point theory tools to show our main results.

To establish Theorem 3.1 we will prove that the functional $\mathcal{I}$ has a global minimum.

Proof of Theorem 3.1. Firstly, we show that $\mathcal{I}$ is coercive, namely, $\mathcal{I}(u) \rightarrow+\infty$ as $\|u\|_{1, \Phi} \rightarrow+\infty$. From (3.2), we have

$$
|F(x, t)| \leq C_{0} \Psi(x, t)+h(x)|t|, \quad \forall(x, t) \in \Omega \times \mathbb{R} .
$$

Then, by applying Lemma 2.1. Poincaré and Hölder's inequalities, and using similar arguments as in the proof of relation (4.7) we obtain

$$
\begin{aligned}
\mathcal{I}(u) & =\int_{\Omega} \Phi(x,|\nabla u|) d x+\int_{\Omega} \frac{V(x)}{q(x)}|u|^{q(x)} d x-\int_{\Omega} F(x, u) d x \\
& \geq\|u\|_{1, \Phi}^{\phi_{0}}-c_{1}\|V\|_{s(x)}\|u\|_{1, \Phi}^{q_{+}}-c_{2}\|u\|_{\Psi}^{\psi^{0}}-c_{3}\|h\|_{\tilde{\Psi}}\|u\|_{\Psi} .
\end{aligned}
$$

Using the fact that $W_{0}^{1, \Phi}(\Omega)$ is compactly embedded in $L^{\Psi}(\Omega)$ (see the proof of relation (4.9)), the previous inequality becomes

$$
\mathcal{I}(u) \geq\|u\|_{1, \Phi}^{\phi_{0}}-c_{1}\|V\|_{s(x)}\|u\|_{1, \Phi}^{q_{+}}-c_{2}^{\prime}\|u\|_{1, \Phi}^{\psi^{0}}-c_{3}^{\prime}\|h\|_{\tilde{\Psi}}\|u\|_{1, \Phi} .
$$

Since $1<q^{+}<\phi_{0}$ and $\psi^{0}<\phi_{0}$, we then have $\mathcal{I}(u) \rightarrow+\infty$ as $\|u\|_{1, \Phi} \rightarrow+\infty$. To complete the proof we show that the functional $\mathcal{I}$ is weakly lower semi-continuous, namely, $u_{n} \rightarrow u$ in $W_{0}^{1, \Phi}(\Omega) \operatorname{implies} \mathcal{I}(u) \leq \liminf _{n \rightarrow \infty} \mathcal{I}\left(u_{n}\right)$. Suppose that $u_{n} \rightarrow u$ in $W_{0}^{1, \Phi}(\Omega)$. Since the functional $\mathcal{H} \in \mathcal{C}^{1}\left(W_{0}^{1, \Phi}(\Omega), \mathbb{R}\right)$ is strictly convex ( $\mathcal{H}^{\prime}$ is strictly monotone), then we have $\mathcal{H}\left(u_{n}\right)>\mathcal{H}(u)+$ $\left\langle\mathcal{H}^{\prime}(u), u_{n}-u\right\rangle$ which implies that $\mathcal{H}$ is weakly lower semi-continuous on $W_{0}^{1, \Phi}(\Omega)$. Concerning the functional $\mathcal{J}$; since $W_{0}^{1, \Phi}(\Omega)$ is compactly embedded in $L^{s^{\prime}(x) q(x)}(\Omega)$ (see the proof of relation (4.5)), then $u_{n} \rightarrow u$ in $L^{s^{\prime}(x) q(x)}(\Omega)$. This fact combined with relation (4.7) yields that $\mathcal{J}\left(u_{n}\right) \rightarrow$ 
$\mathcal{J}(u)$. Finally, from Proposition 4.2 -ii), $\mathcal{F}$ is sequentially weakly continuous. Then, we have $\mathcal{F}\left(u_{n}\right) \rightarrow \mathcal{F}(u)$.

We shall now prove Theorem 3.2 by using the Mountain Pass Theorem (see 2]). Since the proof of this theorem is quite long, we will divide it into several lemmas. Firstly, we show that the functional $\mathcal{I}$ has a geometrical structure. Secondly, we show that $\mathcal{I}$ satisfies the Palais-Smale condition at level $\tilde{c}$ (see the Definition 4.2 below). To this end, we show that any Palais-Smale sequence at the level $\tilde{c}$ for $\mathcal{I}$ (see the Definition 4.1) is bounded in $W_{0}^{1, \Phi}(\Omega)$, and then has a strongly convergent subsequence.

Let us start by the following lemma.

Lemma 4.1. Assume that $\phi^{0}<q^{-} \leq q^{+}<\left(\phi_{0}\right)^{*}, q^{+}-\frac{1}{2} \phi_{0}<q^{-}$and $s(x)>\frac{q(x)\left(\phi_{0}\right)^{*}}{\left(\phi_{0}\right)^{*}-q(x)}$ for every $x \in \bar{\Omega}$. Then, for any function $V \in L^{s(x)}(\Omega)$ we have

$$
\int_{\Omega}\left|V(x)\left\|\left.u\right|^{q(x)} d x \leq C\right\| V \|_{s(x)}^{\frac{\alpha}{r-}}\left[M_{1}+M_{2}\left(\|u\|_{1, \Phi}^{2\left(q^{+}-\theta\right)}+\|u\|_{1, \Phi}^{2 \frac{\theta r^{+}}{r^{-}}}\right)\right],\right.
$$

where $\alpha=r^{+}$if $\|V\|_{s(x)}>1$ and $\alpha=r^{-}$if $\|V\|_{s(x)} \leq 1$, and $2 \frac{\theta r^{+}}{r^{-}}<2\left(q^{-}-\theta\right)<2\left(q^{+}-\theta\right)<\phi_{0}$ for some measurable function $r$ and positive constants $C$ and $\theta$.

Proof. Since we have $q^{+}-\frac{1}{2} \phi_{0}<q^{-}$, then there exists $\theta>0$ such that $q^{+}-\frac{1}{2} \phi_{0}<\theta<q^{-}$. This fact implies that $2\left(q^{-}-\theta\right)<2\left(q^{+}-\theta\right)<\phi_{0}$ and $1+\theta-q^{+}>0$. Let $r$ be any measurable function satisfying,

$$
\begin{aligned}
& \max \left\{\frac{s(x)}{1+\theta s(x)}, \frac{\left(\phi_{0}\right)^{*}}{\left(\phi_{0}\right)^{*}+\theta-q(x)}\right\}<r(x)<\min \left\{\frac{s(x)\left(\phi_{0}\right)^{*}}{\left(\phi_{0}\right)^{*}+\theta s(x)}, \frac{1}{1+\theta-q(x)}\right\}, \\
& \theta\left(\frac{r^{+}}{r^{-}}+1\right)<q^{-}, \quad \forall x \in \Omega .
\end{aligned}
$$

It is clear that $r \in L^{\infty}(\Omega)$ and $1<r(x)<s(x)$. Now, by using Hölder's inequality, we get

$$
\left.\int_{\Omega}\left|V(x)\left\|\left.u\right|^{q(x)} d x \leq C\right\| V\right| u\right|^{\theta}\left\|_{r(x)}\right\||u|^{q(x)-\theta} \|_{(r(x))^{\prime}}
$$

Without loss of generality, we may assume that $\left\|V(x)|u|^{\theta}\right\|_{r(x)}>1$. Using again Hölder's inequality, (2.10), and Proposition 2.3, we obtain

$$
\begin{aligned}
& \left\|V|u|^{\theta}\right\|_{r(x)} \leq\left[\int_{\Omega}|V(x)|^{r(x)}|u|^{\theta r(x)} d x\right]^{\frac{1}{r^{-}}} \\
& \leq C_{1}\left\||V|^{r(x)}\right\|_{\frac{s(x)}{r(x)}}^{\frac{1}{r-}}\left\||u|^{\theta r(x)}\right\|_{\frac{\left(\frac{s(x)}{r(x)}\right)}{r^{-}}}^{\frac{1}{(x)}} \\
& \leq C_{2}\|V\|_{s(x)}^{\frac{\alpha}{r-}}\left(1+\|u\|_{\theta r(x)\left(\frac{s(x)}{r(x)}\right)^{\prime}}^{\frac{\theta r^{\prime}}{r-}}\right),
\end{aligned}
$$

where $\alpha=r^{+}$if $\|V\|_{s(x)}>1$ and $\alpha=r^{-}$if $\|V\|_{s(x)} \leq 1$.

Using the same arguments as above, we obtain

$$
\left\||u|^{q(x)-\theta}\right\|_{(r(x))^{\prime}} \leq 1+\|u\|_{(q(x)-\theta)(r(x))^{\prime}}^{q^{+}-\theta}
$$

Since $r(x)$ is chosen such that (4.13) is fulfilled then

$$
1<\theta r(x)\left(\frac{s(x)}{r(x)}\right)^{\prime}<\left(\phi_{0}\right)^{*} \quad \text { and } \quad 1<(q(x)-\theta)(r(x))^{\prime}<\left(\phi_{0}\right)^{*}, \forall x \in \Omega .
$$

Since $\Phi_{*}$ satisfies $\left(H_{5}\right)$, then by using Lemma 2.3 , we have $|t|^{\theta r(x)\left(\frac{s(x)}{r(x)}\right)^{\prime}} \preccurlyeq \Phi_{*}$ and $|t|^{(q(x)-\theta)(r(x))^{\prime}} \preccurlyeq$ $\Phi_{*}$, which imply that $L^{\Phi_{*}}(\Omega)$ is continuously embedded in $L^{\theta r(x)\left(\frac{s(x)}{r(x)}\right)^{\prime}}(\Omega)$ and in $L^{(q(x)-\theta)(r(x))^{\prime}}(\Omega)$. 
Therefore, from Theorem $2.1 W_{0}^{1, \Phi}(\Omega)$ is continuously embedded in $L^{\theta r(x)\left(\frac{s(x)}{r(x)}\right)^{\prime}}(\Omega)$ and in $L^{(q(x)-\theta)(r(x))^{\prime}}(\Omega)$. Consequently, the relations (4.16) and (4.17) become respectively

$$
\left\|V|u|^{\theta}\right\|_{r(x)} \leq C^{\prime}\|V\|_{s(x)}^{\frac{\alpha}{r-}}\left(1+\|u\|_{1, \Phi}^{\frac{\theta r^{+}}{r^{-}}}\right)
$$

and

$$
\left\||u|^{q(x)-\theta}\right\|_{(r(x))^{\prime}} \leq C^{\prime \prime}\left(1+\|u\|_{1, \Phi}^{q^{+}-\theta}\right) .
$$

Substituting (4.18) and (4.19) into (4.15), and using Young's inequality we obtain

$$
\int_{\Omega}\left|V(x)\left\|\left.u\right|^{q(x)} d x \leq C\right\| V \|_{s(x)}^{\frac{\alpha}{r-}}\left[M_{1}+M_{2}\left(\|u\|_{1, \Phi}^{2\left(q^{+}-\theta\right)}+\|u\|_{1, \Phi}^{2 \frac{\theta^{+}+}{r^{-}}}\right)\right],\right.
$$

where $C, M_{1}$, and $M_{2}$ are positive constants.

Lemma 4.2. Assume that the assumptions $(\Phi),\left(f_{0}^{\prime}\right),\left(f_{2}\right)$ and $\left(f_{3}\right)$ hold. Furthermore, assume that $\phi^{0}<\min \left\{\psi_{0}, q^{-}\right\}, \max \left\{\psi^{0}, q^{+}\right\}<\left(\phi_{0}\right)^{*}, q^{+}-\frac{1}{2} \phi_{0}<q^{-}$, and $s(x)>\frac{q(x)\left(\phi_{0}\right)^{*}}{\left(\phi_{0}\right)^{*}-q(x)}$ for every $x \in \bar{\Omega}$. Then, the functional $\mathcal{I}$ has a geometrical structure, that is, $\mathcal{I}$ satisfies the following properties

(i) there exist $\rho>0$ and $\beta>0$ such that $\mathcal{I}(u) \geq \beta$ for any $u \in W_{0}^{1, \Phi}(\Omega)$ with $\|u\|_{1, \Phi}=\rho$.

(ii) there exists $u_{0} \in W_{0}^{1, \Phi}(\Omega)$ such that $\left\|u_{0}\right\|_{1, \Phi}>\rho$ and $\mathcal{I}\left(u_{0}\right) \leq 0$.

Proof. (i) Firstly, from $\left(f_{0}^{\prime}\right)$ and $\left(f_{3}\right)$ it follows that, for all given $\epsilon>0$ there exists $C(\epsilon)>0$, such that

$$
|F(x, t)| \leq \epsilon \Phi(x, t)+C(\epsilon) \Psi(x, t), \quad \forall(x, t) \in \Omega \times \mathbb{R} .
$$

Using Lemma 2.1. the Poincaré inequality, and the fact that $W_{0}^{1, \Phi}(\Omega)$ is compactly embedded in $L^{\Psi}(\Omega)$, we obtain

$$
\int_{\Omega}|F(x, t)| d x \leq \epsilon \max \left\{\|u\|_{1, \Phi}^{\phi_{0}},\|u\|_{1, \Phi}^{\phi^{0}}\right\}+C^{\prime}(\epsilon) \max \left\{\|u\|_{1, \Phi}^{\psi_{0}},\|u\|_{1, \Phi}^{\psi^{0}}\right\} .
$$

Using the same arguments as in the proof of relation (4.7), we obtain

$$
\int_{\Omega} \frac{V(x)}{q(x)}|u|^{q(x)} \leq C\|V\|_{s(x)} \max \left\{\|u\|_{1, \Phi}^{q_{-}},\|u\|_{1, \Phi}^{q_{+}}\right\} .
$$

Now, by using the definition of $\mathcal{I}$ in (4.1), Lemma 2.1, and the relations (4.22)-(4.23), we get

$$
\begin{aligned}
\mathcal{I}(u)= & \int_{\Omega} \Phi(x,|\nabla u|) d x+\int_{\Omega} \frac{V(x)}{q(x)}|u|^{q(x)} d x-\int_{\Omega} F(x, u) d x \\
\geq & \min \left\{\|u\|_{1, \Phi}^{\phi_{0}},\|u\|_{1, \Phi}^{\phi^{0}}\right\}-C\|V\|_{s(x)} \max \left\{\|u\|_{1, \Phi}^{q_{-}},\|u\|_{1, \Phi}^{q_{+}}\right\} \\
& \quad-\epsilon \max \left\{\|u\|_{1, \Phi}^{\phi_{0}},\|u\|_{1, \Phi}^{\phi^{0}}\right\}-C^{\prime}(\epsilon) \max \left\{\|u\|_{1, \Phi}^{\psi_{0}},\|u\|_{1, \Phi}^{\psi^{0}}\right\},
\end{aligned}
$$

which implies that, for all $u \in W_{0}^{1, \Phi}(\Omega)$ with $\|u\|_{1, \Phi}<1$,

$$
\begin{aligned}
\mathcal{I}(u) & \geq\|u\|_{1, \Phi}^{\phi^{0}}-C\|V\|_{s(x)}\|u\|_{1, \Phi}^{q_{-}}-\epsilon\|u\|_{1, \Phi}^{\phi_{0}}-C^{\prime}(\epsilon)\|u\|_{1, \Phi}^{\psi_{0}} \\
& \geq \frac{1}{2}\|u\|_{1, \Phi}^{\phi^{0}}-C\|V\|_{s(x)}\|u\|_{1, \Phi}^{q_{-}}-C^{\prime}(\epsilon)\|u\|_{1, \Phi}^{\psi_{0}} \\
& =\|u\|_{1, \Phi}^{\phi^{0}}\left(\frac{1}{2}-C\|V\|_{s(x)}\|u\|_{1, \Phi}^{\left(q_{-}\right)-\phi^{0}}-C^{\prime}(\epsilon)\|u\|_{1, \Phi}^{\psi_{0}-\phi^{0}}\right) .
\end{aligned}
$$

Since $\left(q_{-}\right)-\phi^{0}>0$ and $\psi_{0}-\phi^{0}>0$, then from (4.24) we can choose $\beta>0$ and $\rho>0$ such that $\mathcal{I}(u) \geq \beta>0$ for any $u \in W_{0}^{1, \Phi}(\Omega)$ with $\|u\|_{1, \Phi}=\rho$.

(ii) From $\left(f_{2}\right)$, it follows that for any $L>0$ there exists a constant $C_{L}:=C(L)>0$ depending on L, such that

$$
F(x, t) \geq L|t|^{\phi^{0}}-C_{L}, \quad \forall(x, t) \in \Omega \times \mathbb{R} .
$$


Let $w \in W_{0}^{1, \Phi}(\Omega)$ with $w>0$. We take $t>1$ large enough to ensure that $\|t w\|_{1, \Phi}>1$. Then from (4.25) and Lemmas 2.1, 4.1, we have

$$
\begin{aligned}
\mathcal{I}(t w)= & \int_{\Omega} \Phi(x,|t \nabla w|) d x+\int_{\Omega} \frac{V(x)}{q(x)}|t w|^{q(x)} d x-\int_{\Omega} F(x, t w) d x \\
\leq & t^{\phi^{0}}\|w\|_{1, \Phi}^{\phi^{0}}+C\|V\|_{s(x)}^{\frac{\alpha}{r-}}\left[M_{1}+M_{2}\left(t^{2\left(q^{+}-\theta\right)}\|w\|_{1, \Phi}^{2\left(q^{+}-\theta\right)}+t^{2 \frac{\theta r^{+}}{r^{-}}}\|w\|_{1, \Phi}^{2 \frac{\theta r^{+}}{r^{-}}}\right)\right] \\
& \quad-L t^{\phi^{0}} \int_{\Omega}|w|^{\phi^{0}} d x+C_{L}|\Omega| \\
= & t^{\phi^{0}}\left(\|w\|_{1, \Phi}^{\phi^{0}}-L \int_{\Omega}|w|^{\phi^{0}} d x\right) \\
& \quad C\|V\|_{s(x)}^{\frac{\alpha}{r-}}\left[M_{1}+M_{2}\left(t^{2\left(q^{+}-\theta\right)}\|w\|_{1, \Phi}^{2\left(q^{+}-\theta\right)}+t^{2 \frac{\theta r^{+}}{r^{-}}}\|w\|_{1, \Phi}^{2 \frac{\theta r^{+}}{r^{-}}}\right)\right]+C_{L}|\Omega| .
\end{aligned}
$$

By choosing $L>0$ such that $\|w\|_{1, \Phi}^{\phi^{0}}-\left.L \int_{\Omega}|w|\right|^{\phi^{0}} d x<0$ and the fact that $2 \frac{\theta r^{+}}{r^{-}}<2\left(q^{+}-\theta\right)<\phi_{0}$, then we obtain $\mathcal{I}(t w) \rightarrow-\infty$ as $t \rightarrow+\infty$. The proof of this lemma is complete.

Remark 4.1. Note that in the proof of the geometrical structure lemma we do not need any sign condition on the potential $V$.

Now, we define the level at $\tilde{c}$ as follows

$$
\tilde{c}=\inf _{\gamma \in \Gamma} \max _{t \in[0,1]} \mathcal{I}(\gamma(t)),
$$

where $\Gamma=\left\{\gamma \in \mathcal{C}\left([0,1], W_{0}^{1, \Phi}(\Omega)\right): \gamma(0)=0, \gamma(1)=u_{0}\right\}$ is the set of continuous paths joining 0 and $u_{0}$, where $u_{0} \in W_{0}^{1, \Phi}(\Omega)$ is defined in the previous lemma. Let us recall the standard definitions of Palais-Smale sequence at the level $\tilde{c}$ and Palais-Smale condition at the level $\tilde{c}$ for a functional $\mathcal{I} \in \mathcal{C}^{1}(E, \mathbb{R})$, where $E$ is a Banach space.

Definition 4.1. Let $E$ be a Banach space with dual space $E^{*}$ and $\left(u_{n}\right)$ a sequence in $E$. We say that $\left(u_{n}\right)$ is a Palais-Smale sequence at the level $\tilde{c}$ for a functional $\mathcal{I} \in \mathcal{C}^{1}(E, \mathbb{R})$ if

$$
\mathcal{I}\left(u_{n}\right) \rightarrow \tilde{c}, \quad \text { and }\left\|\mathcal{I}^{\prime}\left(u_{n}\right)\right\|_{E^{*}} \rightarrow 0 .
$$

Definition 4.2. We say that a functional $\mathcal{I}$ satisfies the Palais-Smale condition at the level $\tilde{c}$ if any Palais-Smale sequence at the level $\tilde{c}$ for $\mathcal{I}$ possesses a convergent subsequence.

We note that, by Lemma 4.2 the existence of a Palais-Smale sequence at the level $\tilde{c}$ for our functional $\mathcal{I}$ is ensured. This can be observed directly from the proof given in [2].

Now, in order to prove that the functional $\mathcal{I}$ satisfies the Palais-Smale condition, we shall first show that any Palais-Smale sequence for $\mathcal{I}$ is bounded. To this end, we have the following lemma:

Lemma 4.3. Assume that the assumptions $(\Phi)$ and $\left(f_{0}^{\prime}\right)-\left(f_{3}\right)$ hold. Furthermore, assume that $\phi^{0}<\min \left\{\psi_{0}, q^{-}\right\}, \max \left\{\psi^{0}, q^{+}\right\}<\left(\phi_{0}\right)^{*}, q^{+}-\frac{1}{2} \phi_{0}<q^{-}$, and $s(x)>\frac{q(x)\left(\phi_{0}\right)^{*}}{\left(\phi_{0}\right)^{*}-q(x)}$ for every $x \in \bar{\Omega}$. If $V$ has a constant sign a.e. on $\Omega$, then any Palais-Samle sequence at the level $\tilde{c}$ for $\mathcal{I}$ is bounded in $W_{0}^{1, \Phi}(\Omega)$.

Proof. Let $\left(u_{n}\right)$ be a Palais-Smale sequence at the level $\tilde{c}$ for $\mathcal{I}$ in $W_{0}^{1, \Phi}(\Omega)$. We prove by contradiction that $\left(u_{n}\right)$ is bounded in $W_{0}^{1, \Phi}(\Omega)$. Assuming that $\left(u_{n}\right)$ is unbounded in $W_{0}^{1, \Phi}(\Omega)$, that is, $\left\|u_{n}\right\|_{1, \Phi} \rightarrow+\infty$.

Let $v_{n}:=\frac{u_{n}}{\left\|u_{n}\right\|_{1, \Phi}}$. It is clear that $\left(v_{n}\right)$ is bounded in $W_{0}^{1, \Phi}(\Omega)$. Hence, there exists a subsequence denoted again $\left(v_{n}\right)$ such that $v_{n}$ converges weakly to $v$ in $W_{0}^{1, \Phi}(\Omega)$. Since $W_{0}^{1, \Phi}(\Omega)$ is compactly embedded in $L^{\Psi}(\Omega)$ (see the proof of relation (4.9)), thus $v_{n}$ converges strongly to $v$ in $L^{\Psi}(\Omega)$, and then a.e. in $\Omega$. 
Define $\Omega_{\neq}:=\{x \in \Omega:|v(x)| \neq 0\}$. We consider two possible cases: $\left|\Omega_{\neq}\right|=0$ or $\left|\Omega_{\neq}\right|>0$. Firstly, we assume that $\left|\Omega_{\neq}\right|=0$, that is, $v=0$ a.e. in $\Omega$. From the definition of $\mathcal{I}$ in (4.1), Lemma 2.1, and the fact that $\left\|u_{n}\right\|_{1, \Phi} \rightarrow+\infty$, we get

$$
\begin{aligned}
\left\|u_{n}\right\|_{1, \Phi}^{\phi_{0}} & \leq \mathcal{I}\left(u_{n}\right)-\int_{\Omega} \frac{V(x)}{q(x)}\left|u_{n}\right|^{q(x)} d x+\int_{\Omega} F\left(x, u_{n}\right) d x \\
& \leq \mathcal{I}\left(u_{n}\right)+\frac{1}{q^{-}} \int_{\Omega}|V(x)|\left|u_{n}\right|^{q(x)} d x+\int_{\Omega} F\left(x, u_{n}\right) d x
\end{aligned}
$$

which implies that

$$
1 \leq \frac{\mathcal{I}\left(u_{n}\right)}{\left\|u_{n}\right\|_{1, \Phi}^{\phi_{0}}}+\frac{1}{q^{-}\left\|u_{n}\right\|_{1, \Phi}^{\phi_{0}}} \int_{\Omega}\left|V(x) \| u_{n}\right|^{q(x)} d x+\int_{\Omega} \frac{F\left(x, u_{n}\right)}{\left\|u_{n}\right\|_{1, \Phi}^{\phi_{0}}} d x .
$$

Now, we shall show that all terms of the right-hand side of (4.27) tend to zero when $n$ is large enough, which is the desired contradiction. Since $\left(u_{n}\right)$ is a Palais-Smale sequence type, then $\left(\mathcal{I}\left(u_{n}\right)\right)$ is bounded. Hence, the first term of the right-hand side of (4.27) tends to zero as $n$ is large enough. For the second one, from Lemma 4.1 we get

$$
\frac{1}{q^{-}\left\|u_{n}\right\|_{1, \Phi}^{\phi_{0}}} \int_{\Omega}\left|V(x)\left\|\left.u_{n}\right|^{q(x)} d x \leq C\right\| V \|_{s(x)}^{\frac{\alpha}{r^{-}}} \frac{M_{1}+M_{2}\left(\left\|u_{n}\right\|_{1, \Phi}^{2\left(q^{+}-\theta\right)}+\left\|u_{n}\right\|_{1, \Phi}^{2 \frac{\theta r^{+}}{r^{-}}}\right)}{q^{-}\left\|u_{n}\right\|_{1, \Phi}^{\phi_{0}}} .\right.
$$

Since, $2 \frac{\theta r^{+}}{r^{-}}<2\left(q^{-}-\theta\right)<2\left(q^{+}-\theta\right)<\phi_{0}$, then passing to the limit in (4.28), we obtain

$$
\frac{1}{q^{-}\left\|u_{n}\right\|_{1, \Phi}^{\phi_{0}}} \int_{\Omega}\left|V(x) \| u_{n}\right|^{q(x)} d x \rightarrow 0, \text { as } n \rightarrow+\infty .
$$

Hence, the second term tends to zero as $n$ is large enough. For the third term, on the one hand it follows from the definition of $F$ that

$$
\int_{\left\{\left|u_{n}\right| \leq M\right\}} \frac{\left|F\left(x, u_{n}\right)\right|}{\left\|u_{n}\right\|_{1, \Phi}^{\phi_{0}}} d x \leq \frac{C(M)}{\left\|u_{n}\right\|_{1, \Phi}^{\phi_{0}}},
$$

where $C(M)$ is a positive constant depending on $M$ defined in (3.4). On the other hand, by using Hölder's inequality, we get

$$
\begin{aligned}
\int_{\left\{\left|u_{n}\right|>M\right\}} \frac{F\left(x, u_{n}\right)}{\left\|u_{n}\right\|_{1, \Phi}^{\phi_{0}}} d x & =\int_{\left\{\left|u_{n}\right|>M\right\}} \frac{F\left(x, u_{n}\right)}{\left|u_{n}\right|^{\phi_{0}}}\left|v_{n}\right|^{\phi_{0}} d x \\
& \leq 2\left\|\frac{F\left(x, u_{n}\right)}{\left|u_{n}\right|^{\phi_{0}}} \chi_{\left\{\left|u_{n}\right|>M\right\}}\right\|\left\|_{\Gamma}\right\|\left|v_{n}\right|^{\phi_{0}} \chi_{\left\{\left|u_{n}\right|>M\right\}} \|_{\tilde{\Gamma}}
\end{aligned}
$$

Without loss of generality, we may suppose that $\left\|\frac{F\left(x, u_{n}\right)}{\left|u_{n}\right|^{\phi_{0}}} \chi_{\left\{\left|u_{n}\right|>M\right\}}\right\|_{\Gamma}>1$. Then, from Lemma 2.1. we get

$$
\left\|\frac{F\left(x, u_{n}\right)}{\left|u_{n}\right|^{\phi_{0}}} \chi_{\left\{\left|u_{n}\right|>M\right\}}\right\|_{\Gamma} \leq\left[\int_{\left\{\left|u_{n}\right|>M\right\}} \Gamma\left(x, \frac{F\left(x, u_{n}\right)}{\left|u_{n}\right| \phi_{0}}\right) d x\right]^{\frac{1}{\gamma_{0}}}
$$

Hence, it follows from (3.4) that,

$$
\left\|\frac{F\left(x, u_{n}\right)}{\left|u_{n}\right|^{\phi_{0}}} \chi_{\left\{\left|u_{n}\right|>M\right\}}\right\|_{\Gamma} \leq C\left[\int_{\Omega} H\left(x, u_{n}\right) d x\right]^{\frac{1}{\gamma_{0}}}+C^{\prime},
$$

where $C$ and $C^{\prime}$ are positive constants independent of $n$. 
In the case where $V \leq 0$ a.e. on $\Omega$, then from the definition of the functional $\mathcal{I}$ we get

$$
\begin{aligned}
\phi^{0} \mathcal{I}\left(u_{n}\right)-\left\langle\mathcal{I}^{\prime}\left(u_{n}\right), u_{n}\right\rangle & =\int_{\Omega}\left[\phi^{0} \Phi\left(x,\left|\nabla u_{n}\right|\right)-\phi\left(x,\left|\nabla u_{n}\right|\right)\left|\nabla u_{n}\right|^{2}\right] d x \\
& +\int_{\Omega} V(x)\left(\frac{\phi^{0}}{q(x)}-1\right)\left|u_{n}\right|^{q(x)} d x+\int_{\Omega}\left(f\left(x, u_{n}\right) u_{n}-\phi^{0} F\left(x, u_{n}\right)\right) d x
\end{aligned}
$$

From (2.1) and the fact that $\phi^{0}<q^{-} \leq q(x)$, the first and the second terms of the right-hand side of (4.32) are nonnegative. Hence, the relation (4.32) becomes

$$
\phi^{0} \mathcal{I}\left(u_{n}\right)-\left\langle\mathcal{I}^{\prime}\left(u_{n}\right), u_{n}\right\rangle \geq \int_{\Omega} H\left(x, u_{n}\right) d x .
$$

It follows from (4.33) that, $\int_{\Omega} H\left(x, u_{n}\right) d x \leq C$, for $n$ large enough.

Now, in the case where $V \geq 0$ a.e. on $\Omega$, then from the definition of the functional $\mathcal{I}$ we get

$$
\begin{aligned}
q^{+} \mathcal{I}\left(u_{n}\right)-\left\langle\mathcal{I}^{\prime}\left(u_{n}\right), u_{n}\right\rangle & =\int_{\Omega}\left[q^{+} \Phi\left(x,\left|\nabla u_{n}\right|\right)-\phi\left(x,\left|\nabla u_{n}\right|\right)\left|\nabla u_{n}\right|^{2}\right] d x \\
& +\int_{\Omega} V(x)\left(\frac{q^{+}}{q(x)}-1\right)\left|u_{n}\right|^{q(x)} d x+\int_{\Omega} H\left(x, u_{n}\right) d x .
\end{aligned}
$$

Since $q(x) \leq q^{+}$, then following the same arguments as for (4.33), we have also $\int_{\Omega} H\left(x, u_{n}\right) d x \leq C$, for $n$ large enough. This fact combined with relation (4.31) yields

$$
\left\|\frac{F\left(x, u_{n}\right)}{\left|u_{n}\right|^{\phi_{0}}} \chi_{\left\{\left|u_{n}\right|>M\right\}}\right\|_{\Gamma} \leq C, \text { for } n \text { large enough. }
$$

where $C$ is a positive constant independent of $n$. Now, it remains to show that $\left\|\left|v_{n}\right|^{\phi_{0}} \chi_{\left\{\left|u_{n}\right|>M\right\}}\right\|_{\tilde{\Gamma}} \rightarrow$ 0 as $n \rightarrow+\infty$. Let $K(x, t):=\tilde{\Gamma}\left(x,|t|^{\phi_{0}}\right)$. Since $\phi_{0}>1$ and $\tilde{\Gamma} \in N(\Omega)$, then it is clear that $K \in N(\Omega)$. Moreover, since $\Gamma$ satisfies $\left(H_{2}\right)$ then $K$ verifies the assumption (2) of Theorem 2.1 and by Remark 2.3. $K(x, k)$ is bounded for each $k>0$. Using Lemmas 2.1] and 2.3. we get

$$
\lim _{t \rightarrow+\infty} \frac{K(x, k t)}{\Phi_{*}(x, t)} \leq \frac{K(x, k)}{\Phi_{*}(x, 1)} \lim _{t \rightarrow+\infty} \frac{1}{t^{\phi_{0}\left(\gamma_{0}\right)^{\prime}-\left(\phi_{0}\right)^{*}}}
$$

where $\left(\gamma_{0}\right)^{\prime}=\frac{\gamma_{0}}{\gamma_{0}-1}$ is defined as in (3.3). Since $\frac{N}{\phi_{0}}<\gamma_{0}$, then $\phi_{0}\left(\gamma_{0}\right)^{\prime}<\left(\phi_{0}\right)^{*}$. From the last inequality it follows that

$$
\lim _{t \rightarrow+\infty} \frac{K(x, k t)}{\Phi_{*}(x, t)}=0 \text {, uniformly for } x \in \Omega .
$$

Thus, form Theorem 2.1. $W_{0}^{1, \Phi}(\Omega)$ is compactly embedded in $L^{K}(\Omega)$, which implies that

$$
\int_{\Omega} \tilde{\Gamma}\left(x,\left|v_{n}\right|^{\phi_{0}}\right) d x \rightarrow 0, \text { as } n \rightarrow+\infty
$$

Consequently,

$$
\left\|\left|v_{n}\right|^{\phi_{0}} \chi_{\left\{\left|u_{n}\right|>M\right\}}\right\|_{\tilde{\Gamma}} \rightarrow 0, \text { as } n \rightarrow+\infty
$$

Hence, passing to the limit in (4.27) and using (4.29), (4.35) and (4.36), we obtain a contradiction.

Secondly, we assume that $\left|\Omega_{\neq}\right|>0$. Then obviously, $\left|u_{n}\right|=\left|v_{n}\right|\left\|u_{n}\right\|_{1, \Phi} \rightarrow+\infty$ in $\Omega_{\neq}$. Hence, for some positive real $M$ we have $\Omega_{\neq} \subset\left\{x \in \Omega:\left|u_{n}\right| \geq M\right\}$ for $n$ large enough. Using Lemma 2.1] 
we get

$$
\begin{aligned}
& \frac{\mathcal{I}\left(u_{n}\right)}{\left\|u_{n}\right\|_{1, \Phi}^{\phi^{0}}} \leq 1+\frac{1}{q^{-}\left\|u_{n}\right\|_{1, \Phi}^{\phi^{0}}} \int_{\Omega}|V(x)|\left|u_{n}\right|^{q(x)} d x-\int_{\Omega} \frac{F\left(x, u_{n}\right)}{\left\|u_{n}\right\|_{1, \Phi}^{\phi^{0}}} d x \\
&= 1+\frac{1}{q^{-}\left\|u_{n}\right\|_{1, \Phi}^{\phi^{0}}} \int_{\Omega}|V(x)|\left|u_{n}\right|^{q(x)} d x-\int_{\left\{\left|u_{n}\right| \leq M\right\}} \frac{\left|F\left(x, u_{n}\right)\right|}{\left\|u_{n}\right\|_{1, \Phi}^{\phi^{0}}} d x \\
&-\int_{\left\{\left|u_{n}\right|>M\right\}} \frac{F\left(x, u_{n}\right)}{\left|u_{n}\right|^{\phi^{0}}}\left|v_{n}\right|^{\phi^{0}} d x .
\end{aligned}
$$

Now, using relations (4.29), 4.30), assumption $\left(f_{2}\right)$ and Fatou's Lemma, we obtain a contradiction. Hence, $\left(u_{n}\right)$ is bounded in $W_{0}^{1, \Phi}(\Omega)$. The proof of this lemma is complete.

Remark 4.2. The preceding lemma holds true under a slightly weaker assumption than $V$ has a constant sign. Indeed, assume that there exists a constant $\rho$ such that $\phi^{0} \leq \rho \leq q^{+}$and $V(x)\left(\frac{\rho}{q(x)}-1\right) \geq 0$ a.e. on $\Omega$. Then, by taking $H(x, t)=f(x, t) t-\rho F(x, t)$ and following the same arguments as in (4.32)-(4.33), we obtain the previous lemma.

To finish the proof of the Palais-Smale condition for $\mathcal{I}$, we only need to show the following lemma:

Lemma 4.4. Assume that the assumptions of Lemma 4.3 hold. Then, the Palais-Smale sequence at the level $\tilde{c}$ for $\mathcal{I}$ possesses a convergent subsequence.

Proof. Let $\left(u_{n}\right)$ be a Palais-Smale sequence at the level $\tilde{c}$ for $\mathcal{I}$ in $W_{0}^{1, \Phi}(\Omega)$. Then, $\mathcal{I}^{\prime}\left(u_{n}\right) \rightarrow 0$ in $\left(W_{0}^{1, \Phi}(\Omega)\right)^{*}$ and from Lemma 4.3, $\left(u_{n}\right)$ is bounded in $W_{0}^{1, \Phi}(\Omega)$. As $W_{0}^{1, \Phi}(\Omega)$ is reflexive, then there exists a subsequence denoted again $\left(u_{n}\right)$ such that $u_{n}$ converges weakly to $u$ in $W_{0}^{1, \Phi}(\Omega)$. From Proposition 4.2 (i), the mapping $\mathcal{H}^{\prime}$ is of type $\left(S_{+}\right)$. Thus, to conclude the result of this lemma it suffices to show that

$$
\limsup _{n \rightarrow \infty}\left\langle\mathcal{H}^{\prime}\left(u_{n}\right), u_{n}-u\right\rangle \leq 0 .
$$

Indeed, using the definition of $\mathcal{I}^{\prime}$ in Proposition 4.1 we have

$$
\left\langle\mathcal{H}^{\prime}\left(u_{n}\right), u_{n}-u\right\rangle=\left\langle\mathcal{I}^{\prime}\left(u_{n}\right), u_{n}-u\right\rangle+\left\langle\mathcal{F}^{\prime}\left(u_{n}\right), u_{n}-u\right\rangle-\left\langle\mathcal{J}^{\prime}\left(u_{n}\right), u_{n}-u\right\rangle .
$$

It is clear that,

$$
\left\langle\mathcal{I}^{\prime}\left(u_{n}\right), u_{n}-u\right\rangle \rightarrow 0 \text {. }
$$

From Proposition 4.2 (ii), $\mathcal{F}^{\prime}$ is a completely continuous linear operator. Hence,

$$
\left\langle\mathcal{F}^{\prime}\left(u_{n}\right), u_{n}-u\right\rangle \rightarrow 0
$$

Now, it remains to show that $\left\langle\mathcal{J}^{\prime}\left(u_{n}\right), u_{n}-u\right\rangle \rightarrow 0$, that is,

$$
\int_{\Omega} V(x)\left|u_{n}\right|^{q(x)-2} u_{n}\left(u_{n}-u\right) d x \rightarrow 0 .
$$

From the assumptions, we have $1<q(x)<\left(\phi_{0}\right)^{*}$ and $1<\alpha(x)<\left(\phi_{0}\right)^{*}$ for every $x \in \bar{\Omega}$ with $\alpha(x):=\frac{s(x) q(x)}{s(x)-q(x)}$. Then, as in the proof of relation (4.6), the space $W_{0}^{1, \Phi}(\Omega)$ is compactly embedded in $L^{q(x)}(\Omega)$ and in $L^{\alpha(x)}(\Omega)$. Since $\left(u_{n}\right)$ is bounded in $W_{0}^{1, \Phi}(\Omega)$, then $u_{n}$ converges strongly to $u$ in $L^{\alpha(x)}(\Omega)$. Consequently, using Hölder's inequality and Proposition 2.3, then (4.41) holds by the following inequality

$$
\begin{aligned}
\left.\left|\int_{\Omega} V(x)\right| u_{n}\right|^{q(x)-2} u_{n}\left(u_{n}-u\right) d x \mid & \leq C_{0}\|V\|_{s(x)}\left\|\left|u_{n}\right|^{q(x)-1}\right\|_{q^{\prime}(x)}\left\|u_{n}-u\right\|_{\alpha(x)} \\
& \leq C_{1}\|V\|_{s(x)}\left\|u_{n}\right\|_{q(x)}^{\tau}\left\|u_{n}-u\right\|_{\alpha(x)},
\end{aligned}
$$


where $C_{1}$ is a positive constant independent of $n$ and $\tau \in\left\{q^{-}-1, q^{+}-1\right\}$. Finally, it follows from (4.39), (4.40) and (4.41) that (4.37) holds. Hence, since $\mathcal{H}^{\prime}$ is of type $\left(S_{+}\right)$, then $u_{n}$ converges strongly to $u$ in $W_{0}^{1, \Phi}(\Omega)$. The proof of Theorem 3.2 is complete.

Next, as $W_{0}^{1, \Phi}(\Omega)$ is a reflexive and separable Banach space, there exist $\left(e_{j}\right)_{j \in \mathbb{N}^{*}} \subseteq W_{0}^{1, \Phi}(\Omega)$ and $\left(e_{j}^{*}\right)_{j \in \mathbb{N}^{*}} \subseteq\left(W_{0}^{1, \Phi}(\Omega)\right)^{*}$ such that

$$
W_{0}^{1, \Phi}(\Omega)=\overline{\operatorname{span}\left\{e_{j}: j \in \mathbb{N}^{*}\right\}}, \quad\left(W_{0}^{1, \Phi}(\Omega)\right)^{*}=\overline{\operatorname{span}\left\{e_{j}^{*}: j \in \mathbb{N}^{*}\right\}}
$$

and

For $k \in \mathbb{N}^{*}$ denote by

$$
\left\langle e_{i}, e_{j}^{*}\right\rangle= \begin{cases}1, & i=j \\ 0, & i \neq j\end{cases}
$$

$$
X_{j}=\operatorname{span}\left\{e_{j}\right\}, \quad Y_{k}=\oplus_{j=1}^{k} X_{j}, \quad \text { and } \quad Z_{k}=\overline{\oplus_{j=k}^{\infty} X_{j}} .
$$

Proof of Theorem 3.3. We denote by

$$
\beta_{k}:=\sup \left\{\int_{\Omega} \Psi(x,|u|) d x:\|u\|_{1, \Phi}=1, u \in Z_{k}\right\} .
$$

Since $\Psi \ll \Phi_{*}$, then $\lim _{k \rightarrow+\infty} \beta_{k}=0$ (see [27, Lemma 4.3]). Now, we verify the conditions of Fountain theorem. It follows from assumption $\left(f_{4}\right)$ that $\mathcal{F}$ is even, hence the functional $\mathcal{I}$ is even. From Lemmas 4.3 and 4.4. $\mathcal{I}$ satisfies the Palais-Smale condition; hence the condition (3) of Fountain theorem holds. It remains to prove that conditions (1) and (2) in Fountain theorem hold.

(1) By $\left(f_{0}^{\prime}\right)$, it follows that

$$
|F(x, t)| \leq C(\Psi(x, t)+|t|), \quad \forall(x, t) \in \Omega \times \mathbb{R} .
$$

Let $u \in Z_{k}$ with $\|u\|_{1, \Phi}>1$. From the definition of $\mathcal{I}$ in (4.1), Lemmas 2.1, 4.1 and Poincaré's inequality, we obtain

$$
\begin{aligned}
\mathcal{I}(u) & =\int_{\Omega} \Phi(x,|\nabla u|) d x+\int_{\Omega} \frac{V(x)}{q(x)}|u|^{q(x)} d x-\int_{\Omega} F(x, u) d x \\
& \geq\|u\|_{1, \Phi}^{\phi_{0}}-C\|V\|_{s(x)}^{\frac{\alpha}{r-}}\left[M_{1}+M_{2}\left(\|u\|_{1, \Phi}^{2\left(q^{+}-\theta\right)}+\|u\|_{1, \Phi}^{2 \frac{\theta_{r}+}{r-}}\right)\right]-C_{1} \int_{\Omega} \Psi(x,|u|) d x-C_{2}\|u\|_{1, \Phi},
\end{aligned}
$$

where we recall that $\alpha=r^{+}$if $\|V\|_{s(x)}>1$ and $\alpha=r^{-}$if $\|V\|_{s(x)} \leq 1$. Furthermore, from Lemma 2.1, we have

$$
\int_{\Omega} \Psi(x,|u|) d x=\int_{\Omega} \Psi\left(x,\|u\|_{1, \Phi} \frac{|u|}{\|u\|_{1, \Phi}}\right) d x \leq\|u\|_{1, \Phi}^{\psi^{0}} \int_{\Omega} \Psi\left(x, \frac{|u|}{\|u\|_{1, \Phi}}\right) d x .
$$

Using the definition of $\beta_{k}$, the relation (4.43) becomes

$$
\mathcal{I}(u) \geq\|u\|_{1, \Phi}^{\phi_{0}}-C\|V\|_{s(x)}^{\frac{\alpha}{r-}}\left[M_{1}+M_{2}\left(\|u\|_{1, \Phi}^{2\left(q^{+}-\theta\right)}+\|u\|_{1, \Phi}^{2 \frac{\theta r^{+}}{r^{-}}}\right)\right]-C_{1}\|u\|_{1, \Phi}^{\psi^{0}} \beta_{k}-C_{2}\|u\|_{1, \Phi} .
$$

Now, let $u_{k} \in Z_{k}$ with $\|u\|_{1, \Phi}=r_{k}=\left(2 C_{1} \beta_{k}\right)^{\frac{1}{\phi_{0}-\psi^{0}}}$. Since $\phi_{0}<\psi^{0}$ and $\lim _{k \rightarrow+\infty} \beta_{k}=0$, then $r_{k} \rightarrow+\infty$ as $k \rightarrow+\infty$. Thus, we have

$$
\begin{gathered}
\mathcal{I}(u) \geq\left(2 C_{1} \beta_{k}\right)^{\frac{\phi_{0}}{\phi_{0}-\psi^{0}}}-C\|V\|_{s(x)}^{\frac{\alpha}{r-}}\left[M_{1}+M_{2}\left(r_{k}^{2\left(q^{+}-\theta\right)}+r_{k}{ }^{2 \frac{\theta_{r}+}{r^{-}}}\right)\right] \\
-C_{1}\left(2 C_{1} \beta_{k}\right)^{\frac{\psi^{0}}{\phi_{0}-\psi^{0}}} \beta_{k}-C_{2} r_{k},
\end{gathered}
$$




$$
\mathcal{I}(u) \geq \frac{1}{2} r_{k}{ }^{\phi_{0}}-C\|V\|_{s(x)}^{\frac{\alpha}{r-}}\left[M_{1}+M_{2}\left(r_{k}^{2\left(q^{+}-\theta\right)}+r_{k}^{2}{ }^{2 \frac{\theta r^{+}}{r^{-}}}\right)\right]-C_{2} r_{k} .
$$

Since $2 \frac{\theta r^{+}}{r^{-}}<2\left(q^{-}-\theta\right)<2\left(q^{+}-\theta\right)<\phi_{0}$ and $1<\phi_{0}$, then

$$
\inf _{\left\{u \in Z_{k},\|u\|=r_{k}\right\}} \mathcal{I}(u) \rightarrow+\infty \text { as } k \rightarrow+\infty .
$$

(2) Let $w \in Y_{k}$ with $w>0,\|w\|_{1, \Phi}=1$ and $t>1$. Then, from Lemmas 2.1 and 4.25) we have

$$
\begin{aligned}
\mathcal{I}(t w) \leq t^{\phi^{0}} & \left(\|w\|_{1, \Phi}^{\phi^{0}}-L \int_{\Omega}|w|^{\phi^{0}} d x\right) \\
& +C\|V\|_{s(x)}^{\frac{\alpha}{r-}}\left[M_{1}+M_{2}\left(t^{2\left(q^{+}-\theta\right)}\|w\|_{1, \Phi}^{2\left(q^{+}-\theta\right)}+t^{2 \frac{\theta r^{+}}{r^{-}}}\|w\|_{1, \Phi}^{2 \frac{\theta r^{+}}{r^{-}}}\right)\right]+C_{L}|\Omega| .
\end{aligned}
$$

It is clear that we can choose $L>0$ so that $\|w\|_{1, \Phi}^{\phi^{0}}-L \int_{\Omega}|w|^{\phi^{0}} d x<0$. With this fact and since $2 \frac{\theta r^{+}}{r^{-}}<2\left(q^{+}-\theta\right)<\phi_{0}$ then we have $\mathcal{I}(t w) \rightarrow-\infty$ as $t \rightarrow+\infty$. Thus, there exists $\tilde{t}>r_{k}>1$ such that $\mathcal{I}(\tilde{t} w)<0$. By setting $\rho_{k}=\tilde{t}$, then we obtain

$$
\max _{\left\{u \in Y_{k},\|u\|=\rho_{k}\right\}} \mathcal{I}(u) \leq 0 .
$$

The proof of this theorem is complete.

\section{Application}

Let us give an example of function $f$ satisfying the assumptions $\left(f_{0}^{\prime}\right)-\left(f_{3}\right)$ and for which our main Theorems 3.2 and 3.3 hold.

Let us fix $\Phi(x, t)=\frac{1}{p(x)}|t|^{p(x)}$ with $p \in \mathcal{C}^{1-0}(\bar{\Omega})$. Then, the operator $\operatorname{div}(\phi(x,|\nabla u|) \nabla u)$ involved in $(P)$ is the $p(x)$-Laplacian operator, i.e. $\Delta_{p(x)} u:=\operatorname{div}\left(|\nabla u|^{p(x)-2} \nabla u\right)$. In this case, we have $\phi_{0}=p^{-}$and $\phi^{0}=p^{+}$with $1<p^{-} \leq p(x) \leq p^{+}<N$.

In the case where $V \geq 0$ a.e. on $\Omega$, we take $F(x, t)=|t|^{q^{+}} \ln (1+|t|)$, with $q^{+}+1<\frac{N p^{-}}{N-p^{-}}$. The derivative with respect to $t$ of $F(x, t)$ is given by $F^{\prime}(x, t):=f(x, t)=q^{+}|t|^{q^{+}-2} t \ln (1+|t|)+\frac{t|t|^{q^{+}-1}}{1+|t|}$ and we have: $f(x, t) t-q^{+} F(x, t)=\frac{|t|^{q^{+}}}{1+|t|}$. It is clear that $f$ satisfies the assumptions $\left(f_{0}^{\prime}\right),\left(f_{2}\right)-\left(f_{4}\right)$. Moreover, since $\frac{F(x, t)}{|t|^{\theta}} \rightarrow 0$ for all $\theta>q^{+}$then from (1.1), $f$ does not satisfy the (A-R) condition. Now, it remains to show that the assumption $\left(f_{1}\right)$ holds. To this end, let us consider the function $\Gamma(x, t)=|t|^{\beta}$, where $1<\frac{N}{p^{-}}<\beta<\frac{q^{+}}{q^{+}-p^{-}}$. Then, $\Gamma\left(x, \frac{F(x, t)}{|t|^{-}}\right)=|t|^{\beta\left(q^{+}-p^{-}\right)} \ln ^{\beta}(1+|t|)$. Since $\beta\left(q^{+}-p^{-}\right)<q^{+}$, then $\frac{|t|^{\beta\left(q^{+}-p^{-}\right)+1} \ln ^{\beta}(1+|t|)}{|t|^{q^{+}+1}} \rightarrow 0$ as $|t| \rightarrow+\infty$. Hence, the assumption $\left(f_{1}\right)$ holds.

Now, in the case where $V \leq 0$ a.e. on $\Omega$ we can take $F(x, t)=|t|^{p^{+}} \ln (1+|t|)$. By the same arguments above, the choice of $\Gamma(x, t)=|t|^{\beta}$, where $1<\frac{N}{p^{-}}<\beta<\frac{p^{+}}{p^{+}-p^{-}}$, ensures easily that $f$ verifies the assumptions $\left(f_{0}^{\prime}\right)-\left(f_{4}\right)$. Consequently, the main Theorems 3.2 and 3.3 hold.

\section{Remark 5.1.}

(1) In the case where $V \leq 0$ a.e. on $\Omega$, we can not take the same function $F$ considered in the first case, i.e. $F(x, t)=|t|^{q^{+}} \ln (1+|t|)$. Indeed, in this case, the nonlinearity $f$ satisfies the $(A-R)$ condition.

(2) As in the first remark, we can not consider the function $F(x, t)=|t|^{p^{+}} \ln (1+|t|)$ when $V \geq 0$ a.e. on $\Omega$. Indeed, in this case we have

$f(x, t) t-q^{+} F(x, t)=\left(p^{+}-q^{+}\right)|t|^{p^{+}} \ln (1+|t|)+\frac{|t|^{p^{+}+1}}{1+|t|}<0$, for $|t|$ large enough.

Hence, the nonlinearity $f$ do not satisfy the assumption $\left(f_{1}\right)$. 


\section{REFERENCES}

[1] A. Abdou, A. Marcos, Existence and multiplicity of solutions for a Dirichlet problem involving perturbed $p(x)$ Laplacian operator, Electron. J. Differential Equations, Vol. 2016 (2016), No. 197, 1-19.

[2] A. Ambrosetti, P. H. Rabinowitz, Dual Variational Methods In Critical Point Theory And Applications, J. Funct. Anal. Vol. 14 (1973), No. 4, 349-381.

[3] N. Benouhiba, On the eigenvalues of weighted $p(x)$-Laplacian on $\mathbb{R}^{N}$, Nonlinear. Anal. Vol. 74 (2011), No. 1, 235-243.

[4] M.L.M. Carvalho, J.V.A. Goncalves, E.D. da Silva, On quasilinear elliptic problems without the AmbrosettiRabinowitz condition, J. Math. Anal. Appl. Vol. 426 (2015), No. 1, 466-483.

[5] G. Bonanno, G. Molica Bisci, V. Rădulescu, Quasilinear elliptic non-homogeneous Dirichlet problems through Orlicz-Sobolev spaces, Nonlinear Anal. Vol. 75 (2012), No. 12, 4441-4456.

[6] G. Bonanno, G. Molica Bisci, V. Rădulescu, Arbitrarily small weak solutions for a nonlinear eigenvalue problem in Orlicz-Sobolev spaces, Monatsh. Math. 165 (3-4), 305-318 (2011).

[7] G. Bonanno, G. Molica Bisci, V. Rădulescu, Infinitely many solutions for a class of nonlinear eigenvalue problems in Orlicz-Sobolev spaces, C.R. Acad. Sci. Paris 349, 263-268 (2011).

[8] G. Bonanno, G. Molica Bisci, V. Rădulescu, Existence of three solutions for a non-homogeneous Neumann problem through Orlicz-Sobolev spaces, Nonlinear Anal. Vol. 74 (2011), No. 14, 4785-4795.

[9] M. Boreanu, P. Pucci, V. Rădulescu, Multiplicity of solutions for a class of anisotropic elliptic equations with variable exponent, Complex Var. Elliptic Equ. 56 (2011), 755-767.

[10] Y. Chen, S. Levine, R. Rao, Variable exponent, linear growth functionals in image restoration, SIAM J. Appl. Math. Vol. 66 (2006), No. 4, 1383-1406.

[11] N.T. Chung, H.Q. Toan, On a nonlinear and non-homogeneous problem without (A-R) type condition in Orlicz-Sobolev spaces, Appl. Math. Comput. Vol. 219 (2013), No. 14, 7820-7829.

[12] Ph. Clément, M. García-Huidobro, R. Manásevich, K. Schmitt, Mountain pass type solutions for quasilinear elliptic equations, Calc. Var. Partial Differential Equations, Vol. 11 (2000), No. 1, 33-62.

[13] D.G. Costa, C.A. Magalhães, Variational elliptic problems which are nonquadratic at infinity, Nonlinear Anal. Vol. 23 (1994), No. 11, 1401-1412.

[14] D. Edmunds, J. Rákosník, Sobolev embeddings with variable exponent, Studia Mathematica, Vol. 143 (2000), No. 3, 267-293.

[15] X. Fan, An imbedding theorem for Musielak-Sobolev spaces, Nonlinear Anal. Vol. 75 (2012), No. 4, $1959-1971$.

[16] X. Fan, Differential equations of divergence form in Musielak-Sobolev spaces and a sub-supersolution method, J. Math. Anal. Appl. Vol. 386 (2012), No. 2, 593-604.

[17] X. Fan, Q. H. Zhang, Existence of solutions for $p(x)$-Laplacian Dirichlet problem, Nonlinear Anal. Vol. 52 (2003), No. 8, 1843-1852.

[18] X. Fan, D. Zhao, On the spaces $L^{p(x)}(\Omega)$ and $W^{1, p(x)}(\Omega)$, J. Math. Anal. Appl. Vol. 263 (2001), No. 2, 424-446.

[19] N. Fukagai, M. Ito, K. Narukawa, Positive solutions of quasilinear elliptic equations with critical Orlicz-Sobolev nonlinearity on $\mathbb{R}^{N}$, Funkcial. Ekvac. Vol. 49 (2006), No. 2, 235-267.

[20] B. Ge, Z.Y. Chen, Existence of infinitely many solutions for double phase problem with sign-changing potential, RACSAM Rev. R. Acad. Cienc. Exactas Fís. Nat. Ser. A Mat. (2019), 1-12.

[21] B. Ge, D.-J. Lv, J.-F. Lua, Multiple solutions for a class of double phase problem without the AmbrosettiRabinowitz conditions, Nonlinear Anal. Vol. 188 (2019), 294-315.

[22] H. Hudzik, On generalized Orlicz-Sobolev space, Funct. Approx. Comment. Math. 1976.

[23] K. Kefi, p(x)-Laplacian with indefinite weight, Proc. Amer. Math. Soc. Vol. 139, (2011), No. 12, 435-4360.

[24] I.H. Kim, Y.H. Kim, Mountain pass type solutions and positivity of the infimum eigenvalue for quasilinear elliptic equations with variable exponents, Manuscripta Math. 147 (2015), 169-191.

[25] O. Kovacik, J. Rakosuik, On spaces $L^{p(x)}(\Omega)$ and $W^{k, p(x)}(\Omega)$, Czechoslovak Math. J. Vol. 41 (1991), No. 4, 592-618.

[26] G. Li, V. Rădulescu, D. D. Repovš, Q. Zhang, Nonhomogeneous Dirichlet problems without the AmbrosettiRabinowitz condition, Topol. Methods Nonlinear Anal. Vol. 51 (2018), No. 1 (2018), 55-77.

[27] D. Liu, P. Zhao, Solutions for a quasilinear elliptic equation in Musielak-Sobolev spaces, Nonlinear. Anal. Vol. 26 (2015), 315-329.

[28] M. Mihăilescu, P. Pucci, V. Rădulescu, Nonhomogeneous boundary value problems in anisotropic Sobolev spaces, C. R. Acad. Sci. Paris, Ser. I 345 (2007), 561-566.

[29] M. Mihăilescu, P. Pucci, V. Rădulescu, Eigenvalue problems for anisotropic quasilinear elliptic equations with variable exponent, J. Math. Anal. Appl. 340 (2008), 687-698.

[30] M. Mihăilescu, V. Rădulescu, Neumann problems associated to non-homogeneous differential operators in Orlicz-Sobolev spaces, Ann. Inst. Fourier, Vol. 58 (2008), No. 6, 2087-2111.

[31] M. Mihăilescu, V. Rădulescu, D. Repovš, On a non-homogeneous eigenvalue problem involving a potential: an Orlicz-Sobolev space setting, J. Math. Pures Appl. Vol. 93 (2010), No. 2, 132-148.

[32] J. Musielak, Orlicz Spaces and Modular Spaces, Lecture Notes in Math. Vol. 1034, Spring-Verlag, Berlin, 1983. 
[33] M.N. Rao, Z.D. Ren, Theory of Orlicz Spaces, Marcel Dekker, New York, 1985.

[34] M. Růžička, Electrorheological Fluids: Modeling and Mathematical Theory, Lecture Notes in Math. Vol. 1748, Springer-Verlag, Berlin, 2000.

[35] V. Rădulescu, Q. Zhang, Double phase anisotropic variational problems and combined effects of reaction and absorption terms, J. Math. Pures Appl. Vol. 118 (2018), 159-203.

[36] B. Wang, D. Liu, P. Zhao, Hölder continuity for nonlinear elliptic problem in Musielak-Orlicz-Sobolev space, J. Differential Equations, Vol. 266 (2019), No. 8, 4835-4863.

[37] M. Willem, Minimax Theorems, Birkhäuser, Boston, 1996.

E-mail address: sf.maatouk@gmail.com

E-mail address: aelhachi@yahoo.fr

Center of Mathematical Research and Applications of Rabat (CeReMaR), Laboratory of Mathematical Analysis and Applications (lama), Department of Mathematics, Faculty of Sciences, Mohammed V University, P.O. Box 1014, Rabat, Morocco. 